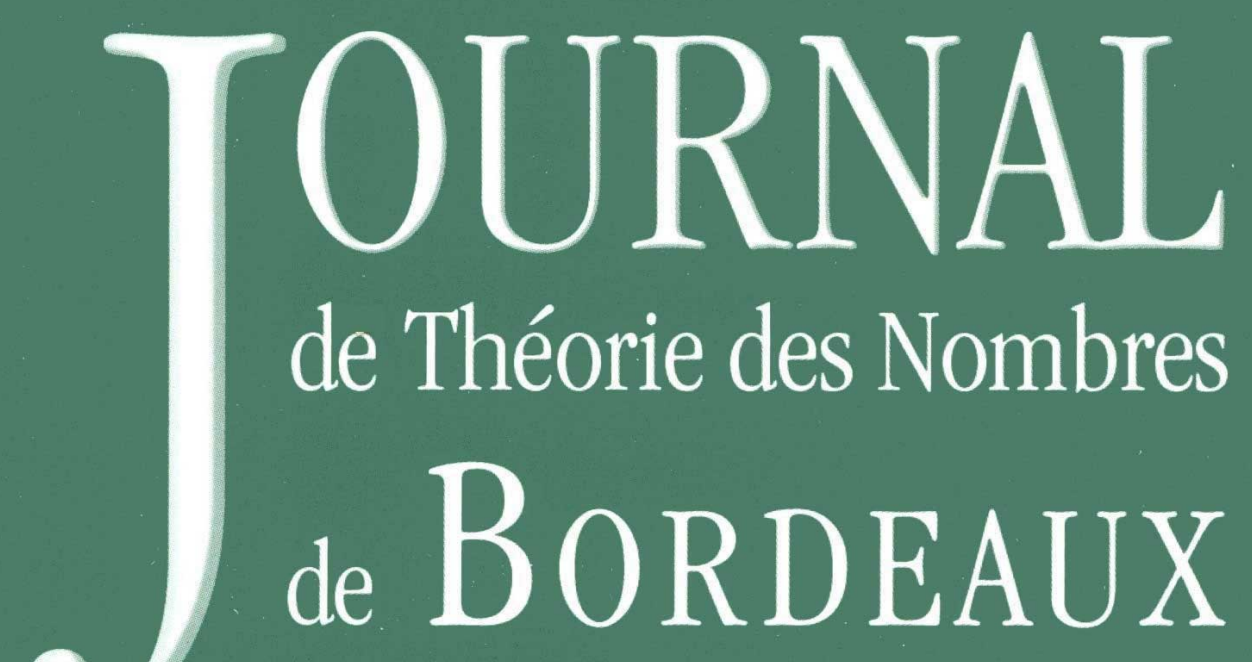

anciennement Séminaire de Théorie des Nombres de Bordeaux

Peter KIRSCHENHOFER, Attila PETHŐ, Paul SURER et Jörg THUSWALDNER

\title{
Finite and periodic orbits of shift radix systems
}

Tome 22, no 2 (2010), p. 421-448.

<http://jtnb.cedram.org/item?id=JTNB_2010__22_2_421_0>

(C) Université Bordeaux 1, 2010, tous droits réservés.

L'accès aux articles de la revue «Journal de Théorie des Nombres de Bordeaux » (http://jtnb.cedram.org/), implique l'accord avec les conditions générales d'utilisation (http://jtnb.cedram. org/legal/). Toute reproduction en tout ou partie cet article sous quelque forme que ce soit pour tout usage autre que l'utilisation à fin strictement personnelle du copiste est constitutive d'une infraction pénale. Toute copie ou impression de ce fichier doit contenir la présente mention de copyright.

\section{cedram}




\title{
Finite and periodic orbits of shift radix systems
}

\author{
par Peter KIRSChenhofer, Attila PethÖ, Paul SURER et Jörg \\ THUSWALDNER
}

\section{Dedicated to the memory of Edmund Hlawka (1916-2009)}

RÉsumé. Pour $\mathbf{r}=\left(r_{0}, \ldots, r_{d-1}\right) \in \mathbb{R}^{d}$, nous définissons la fonction

$$
\tau_{\mathbf{r}}: \mathbb{Z}^{d} \rightarrow \mathbb{Z}^{d}, \quad \mathbf{z}=\left(z_{0}, \ldots, z_{d-1}\right) \mapsto\left(z_{1}, \ldots, z_{d-1},-\lfloor\mathbf{r z}\rfloor\right),
$$

où $\mathbf{r z}$ est le produit scalaire des vecteurs $\mathbf{r}$ et $\mathbf{z}$. Si chaque orbite de $\tau_{\mathbf{r}}$ se termine par $\mathbf{0}$, nous dirons que $\tau_{\mathbf{r}}$ est un shift radix system. Il est bien connu que chaque orbite de $\tau_{\mathbf{r}}$ est ultimement périodique si le polynôme $t^{d}+r_{d-1} t^{d-1}+\cdots+r_{0}$ associé à $\mathbf{r}$ est contractant. D'autre part, si ce polynôme a au moins une racine en dehors du disque unité, il existe des vecteurs initiaux qui conduisent à des orbites non-bornées. Le présent article considère les cas restants pour les propriétés de périodicité des applications $\tau_{\mathbf{r}}$ pour des vecteurs $\mathbf{r}$ associés à des polynômes dont les racines ont un module supérieur ou égal à un, avec égalité dans au moins un cas. Nous montrons que pour une large classe de vecteurs $\mathbf{r}$ appartenant à la famille précédente, l'ultime périodicité des orbites est équivalente au fait que $\tau_{\mathbf{s}}$ est un shift radix system ou a une autre structure prescrite d'orbite pour un certain paramètre $\mathbf{s}$ dépendant de $\mathbf{r}$. Ces résultats sont combinés avec de nouveaux résultats algorithmiques dans le but de caractériser les vecteurs $\mathbf{r}$ de la classe précédente qui donnent des orbites ultimement périodiques pour chaque valeur initiale. En particulier, nous donnons la description de ces vecteurs $\mathbf{r}$ pour le cas $d=3$. Cela conduit à des ensembles qui semblent avoir une structure très compliquée.

Manuscrit reçu le 15 juin 2009.

The first and fourth author were supported by the Austrian Science Foundation (FWF), project S9610, which is part of the national research network FWF-S96 "Analytic combinatorics and probabilistic number theory".

The second author was supported partially by the project JP-26/2006 and by the Hungarian National Foundation for Scientific Research Grant No. T67580.

The third author was supported by the Austrian Science Foundation (FWF), project S9610, which is part of the national research network FWF-S96 "Analytic combinatorics and probabilistic number theory" and by the brazilian FAPESP, Proc. 2009/07744-0.

Mots clefs. Contractive polynomial, shift radix system, periodic orbit.

Classification math.. 11A63. 
Abstract. For $\mathbf{r}=\left(r_{0}, \ldots, r_{d-1}\right) \in \mathbb{R}^{d}$ define the function

$$
\tau_{\mathbf{r}}: \mathbb{Z}^{d} \rightarrow \mathbb{Z}^{d}, \quad \mathbf{z}=\left(z_{0}, \ldots, z_{d-1}\right) \mapsto\left(z_{1}, \ldots, z_{d-1},-\lfloor\mathbf{r z}\rfloor\right),
$$

where $\mathbf{r z}$ is the scalar product of the vectors $\mathbf{r}$ and $\mathbf{z}$. If each orbit of $\tau_{\mathbf{r}}$ ends up at $\mathbf{0}$, we call $\tau_{\mathbf{r}}$ a shift radix system. It is a well-known fact that each orbit of $\tau_{\mathbf{r}}$ ends up periodically if the polynomial $t^{d}+r_{d-1} t^{d-1}+\cdots+r_{0}$ associated to $\mathbf{r}$ is contractive. On the other hand, whenever this polynomial has at least one root outside the unit disc, there exist starting vectors that give rise to unbounded orbits. The present paper deals with the remaining situations of periodicity properties of the mappings $\tau_{\mathbf{r}}$ for vectors $\mathbf{r}$ associated to polynomials whose roots have modulus less than or equal to one with equality in at least one case. We show that for a large class of vectors $\mathbf{r}$ belonging to the above class the ultimate periodicity of the orbits of $\tau_{\mathbf{r}}$ is equivalent to the fact that $\tau_{\mathbf{s}}$ is a shift radix system or has another prescribed orbit structure for a certain parameter $\mathbf{s}$ related to $\mathbf{r}$. These results are combined with new algorithmic results in order to characterize vectors $\mathbf{r}$ of the above class that give rise to ultimately periodic orbits of $\tau_{\mathbf{r}}$ for each starting value. In particular, we work out the description of these vectors $\mathbf{r}$ for the case $d=3$. This leads to sets which seem to have a very intricate structure.

\section{Introduction}

Shift radix systems were introduced in 2005 by Akiyama et al. [1] in the following way. For $\mathbf{r}=\left(r_{0}, \ldots, r_{d-1}\right) \in \mathbb{R}^{d}$ define the function

$$
\tau_{\mathbf{r}}: \mathbb{Z}^{d} \rightarrow \mathbb{Z}^{d}, \quad \mathbf{z}=\left(z_{0}, \ldots, z_{d-1}\right) \mapsto\left(z_{1}, \ldots, z_{d-1},-\lfloor\mathbf{r z}\rfloor\right),
$$

where $\mathbf{r z}$ is the scalar product of the vectors $\mathbf{r}$ and $\mathbf{z}$. Note that $\tau_{\mathbf{r}}(\mathbf{z})=$ $\left(z_{1}, \ldots, z_{d}\right)$ where $z_{d}$ is defined uniquely by

$$
0 \leq r_{0} z_{0}+\cdots+r_{d-1} z_{d-1}+z_{d}<1 .
$$

This relates the study of $\tau_{\mathbf{r}}$ to almost linear recurrences (see e.g. $[2,3,12]$ where this viewpoint is emphasized).

The mapping $\tau_{\mathbf{r}}$ is called a shift radix system (SRS) if for any $\mathbf{z} \in \mathbb{Z}^{d}$ there exists $k \in \mathbb{N}$ such that ${ }^{1} \tau_{\mathbf{r}}^{k}(\mathbf{z})=\mathbf{0}$. Shift radix systems turned out to be a generalization of several notions of well-known number systems. For certain parameters $\mathbf{r}$ related to a Pisot number $\beta$ the mapping $\tau_{\mathbf{r}}$ is conjugate to the well-known beta-transformation $T_{\beta}(\gamma)=\beta \gamma-\lfloor\beta \gamma\rfloor$. This conjugacy can be used in order to prove that the beta-expansions related to $\beta$ have a certain finiteness property (called property $(F)$ in the literature; cf. e.g. [10]) if and only if the related mapping $\tau_{\mathbf{r}}$ is an SRS. Moreover, the problem of characterizing all bases of canonical number systems (see [15] for

\footnotetext{
$1_{\text {The notation }} \tau_{\mathbf{r}}^{k}(\mathbf{z})$ denotes the $k$ th iterate of $\tau_{\mathbf{r}}$ applied to $\mathbf{z}$.
} 
a definition of these objects) turns out to be a special case of the description of all vectors $\mathbf{r}$ giving rise to SRS. For details of these correspondences we refer the reader to [1]. It is also possible to view the rational based number systems introduced in [5] as special cases of SRS.

SRS are related to orbits of $\tau_{\mathbf{r}}$ ending up eventually at zero. As we are interested in eventually periodic orbits of $\tau_{\mathbf{r}}$ in general, we define the sets

$$
\begin{aligned}
\mathcal{D}_{d} & :=\left\{\mathbf{r} \in \mathbb{R}^{d} \mid \forall \mathbf{z} \in \mathbb{Z}^{d} \exists k, l \in \mathbb{N}: \tau_{\mathbf{r}}^{k}(\mathbf{z})=\tau_{\mathbf{r}}^{k+l}(\mathbf{z})\right\} \quad \text { and } \\
\mathcal{D}_{d}^{(0)} & :=\left\{\mathbf{r} \in \mathbb{R}^{d} \mid \tau_{\mathbf{r}} \text { is an SRS }\right\}
\end{aligned}
$$

which will be of importance throughout the present paper. In view of the above-mentioned facts the set $\mathcal{D}_{d}^{(0)}$ is related to finiteness properties of several kinds of number systems. The set $\mathcal{D}_{d}$ is strongly related to contractive polynomials. Indeed, for $\mathbf{r}=\left(r_{0}, \ldots, r_{d-1}\right) \in \mathbb{R}^{d}$ define

$$
R(\mathbf{r}):=\left(\begin{array}{ccccc}
0 & 1 & 0 & \cdots & 0 \\
\vdots & 0 & \ddots & \ddots & \vdots \\
\vdots & \vdots & \ddots & 1 & 0 \\
0 & 0 & \cdots & 0 & 1 \\
-r_{0} & -r_{1} & \cdots & -r_{d-2} & -r_{d-1}
\end{array}\right) \in \mathbb{R}^{d \times d}
$$

and let

$$
\mathcal{E}_{d}:=\left\{\mathbf{r} \in \mathbb{R}^{d} \mid \varrho(R(\mathbf{r}))<1\right\}
$$

where $\varrho(A)$ denotes the spectral radius of the square matrix $A$. It is easy to see that $\mathcal{E}_{d}$ is the so-called Schur-Cohn-region (see [18]) which contains all vectors $\mathbf{r}$ for which $\mathbf{r} \cdot\left(x^{0}, x^{1}, \ldots, x^{d-1}\right)$ is a contractive polynomial in $x$. In $[1$, Section 4$]$ it is shown that $\mathcal{D}_{d}$ satisfies

$$
\mathcal{E}_{d} \subset \mathcal{D}_{d} \subset \overline{\mathcal{E}_{d}}
$$

and $\mathcal{E}_{d}=\operatorname{int}\left(\overline{\mathcal{E}_{d}}\right)$. We mention here that the set $\mathcal{E}_{d}$ was discussed in [9], where it was shown that it is simply connected and that it is bounded by three hypersurfaces two of which are hyperplanes for $d \geq 2$.

The only problem remaining for the characterization of $\mathcal{D}_{d}$ is to describe $\mathcal{D}_{d} \backslash \mathcal{E}_{d}$, which is a subset of $\partial \mathcal{D}_{d}$. We just mention that this problem contains a well-known conjecture on Salem numbers as a special case. Indeed, in 1980 Schmidt [17] conjectured that beta-expansions with respect to a Salem number $\beta$ are periodic for each $\gamma \in \mathbb{Q}(\beta)$. It turns out that this is equivalent to the fact that the mapping $\tau_{\mathbf{r}}$ has only eventually periodic orbits, i.e., $\mathbf{r} \in \mathcal{D}_{d}$, for a certain $\mathbf{r}$ related to $\beta$. As $\beta$ is a Salem number this parameter $\mathbf{r}$ is contained in $\partial \mathcal{D}_{d}$. Some studies related to the original conjecture of Schmidt can be found in [6], however, it has not been settled for a single Salem number up to now. Although in the present paper we are able to 

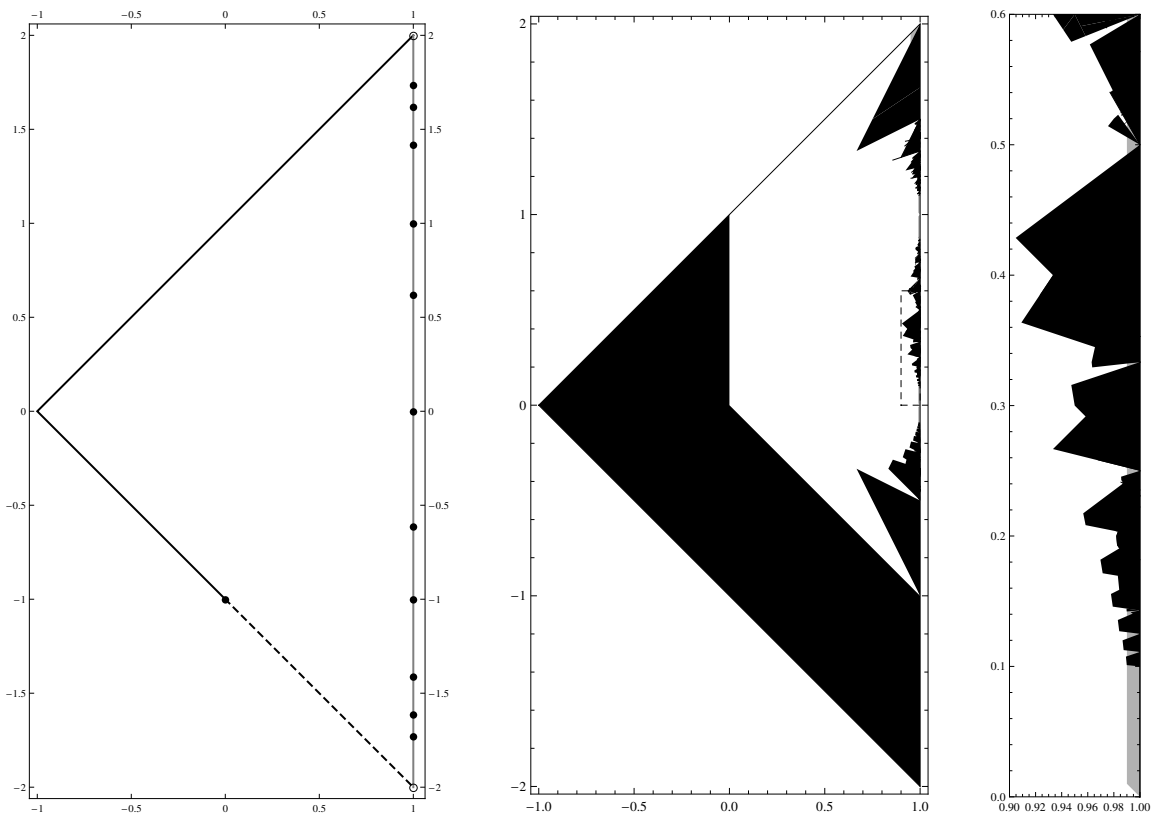

Figure 1.1. The set $\mathcal{D}_{2}$ (left) and an approximation of $\mathcal{D}_{2}^{(0)}$ (middle). On the right hand side we give a magnified version of the dashed rectangle indicated in the approximation of $\mathcal{D}_{2}^{(0)}$. In this magnification some parts of the small grey region which is not yet characterized is visible.

describe large parts of $\mathcal{D}_{d} \backslash \mathcal{E}_{d}$ we cannot settle Schmidt's conjecture. Our parameter classes also do not contain Salem numbers.

For the more general problem of describing $\mathcal{D}_{d} \backslash \mathcal{E}_{d}$ the situation is a bit different. Indeed, there exist some subsets of $\partial \mathcal{D}_{d}$ (not related to Salem numbers) which could be characterized so far. Partial results for $d=2$ can be found in $[2,3,4,14]$. Moreover, in [16] several regions of $\partial \mathcal{D}_{3}$ have been investigated. The current situation for $d=2$ is depicted on the left hand side of Figure 1.1. The interior of the triangle represents $\mathcal{E}_{2}$. The solid lines and the points marked with small full disks form a subset of $\mathcal{D}_{2}$ while the dashed lines together with the points marked with small circles $((1,2)$ and $(1,-2))$ do not. It is up to now unknown whether the grey parts of the right boundary of the triangle belong to $\mathcal{D}_{2}$ or not. It is conjectured that they do (see [4, Conjecture 6.1]). For the three dimensional case there exists only a case study (see [12]). More precisely, it was shown that $\left(1, \frac{3+\sqrt{5}}{2}, \frac{3+\sqrt{5}}{2}\right) \in$ $\partial \mathcal{D}_{3} \backslash \mathcal{D}_{3}$. Summing up, it seems to be very difficult to describe the boundary of $\mathcal{D}_{d}$ and often even for a single point it seems to be hard to decide whether it belongs to $\mathcal{D}_{d}$ or not. 
The aim of the present paper is to give a new method which allows to decide for large regions of $\partial \mathcal{D}_{d}$ whether they belong to $\mathcal{D}_{d}$ or not.

Our method works by establishing connections between the sets $\mathcal{D}_{d}$ and $\mathcal{D}_{e}^{(0)}$ (where $e<d$ ) as well as some related sets. Knowledge on the sets $\mathcal{D}_{e}^{(0)}$ is used in order to make assertions on the boundary of $\mathcal{D}_{d}$. Although the sets $\mathcal{D}_{d}^{(0)}$ are far from being described completely, large regions of them can be described algorithmically which is a priori not possible for points in $\mathcal{D}_{d} \backslash \mathcal{E}_{d}$. Indeed, characterization results on $\mathcal{D}_{d}^{(0)}$ can be found in $[1,4]$. Moreover, a very good approximation of $\mathcal{D}_{2}^{(0)}$ is contained in [19]. This approximation is shown in the middle of Figure 1.1. The white parts inside the triangle belong to $\mathcal{D}_{2}^{(0)}$, the black parts do not. The small grey sets near the right boundary that can be seen in the magnification on the right hand side of Figure 1.1 have not been analyzed yet. The only complete result available so far is for $d=1$. Here we have $\mathcal{D}_{1}=[-1,1]$ and $\mathcal{D}_{1}^{(0)}=[0,1)(c f .[1$, Proposition 4.4]).

The paper is organized as follows. In Section 2 we collect several properties of $\mathcal{E}_{d}$ which will be used in the sequel. For this purpose we summarize and adapt the results of Fam and Meditch [9]. Section 3 contains first results on the orbit structure of $\tau_{\mathbf{r}}$. In Section 4 we define sets determined by certain cycles of $\tau_{\mathbf{r}}$ that will be relevant in the sequel. Section 5 contains our main results. We give characterization results for large parts of $\mathcal{D}_{d} \backslash \mathcal{E}_{d}$ in terms of sets which can be determined algorithmically. In particular, we relate some parts of $\mathcal{D}_{d} \backslash \mathcal{E}_{d}$ to $\mathcal{D}_{d-1}^{(0)}, \mathcal{D}_{d-2}^{(0)}$ as well as some related sets large parts of which can be described algorithmically. The results are based on a fairly general theorem on the orbit structure of $\tau_{\mathbf{r}}$. Section 6 is devoted to algorithms for determining the relevant sets. Using these algorithms we give a description of large parts of $\mathcal{D}_{3} \backslash \mathcal{E}_{3}$, compare Figures 6.1 and 6.2.

\section{Some properties of $\mathcal{E}_{d}$}

The set $\mathcal{E}_{d}$ can be described by using a result of Schur [18]. 


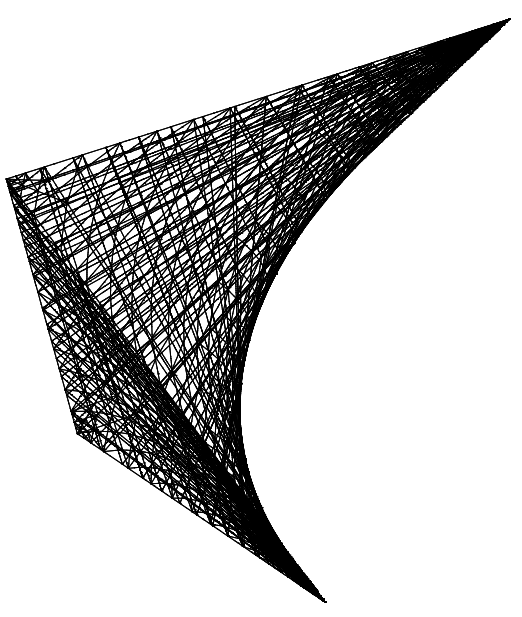

Figure 2.1. The set $\mathcal{E}_{3}$

Proposition 2.1 (cf. Schur [18]). For $0 \leq k<d$ define $\delta_{k}\left(r_{0}, \ldots, r_{d-1}\right) \in$ $\mathbb{R}^{2(k+1) \times 2(k+1)}$ by

$$
\delta_{k}\left(r_{0}, \ldots, r_{d-1}\right)=\left(\begin{array}{cccccccc}
1 & 0 & \cdots & 0 & r_{0} & \cdots & \cdots & r_{k} \\
r_{d-1} & \ddots & \ddots & \vdots & 0 & \ddots & & \vdots \\
\vdots & \ddots & \ddots & 0 & \vdots & \ddots & \ddots & \vdots \\
r_{d-k-1} & \cdots & r_{d-1} & 1 & 0 & \cdots & 0 & r_{0} \\
r_{0} & 0 & \cdots & 0 & 1 & r_{d-1} & \cdots & r_{d-k-1} \\
\vdots & \ddots & \ddots & \vdots & 0 & \ddots & \ddots & \vdots \\
\vdots & & \ddots & 0 & \vdots & \ddots & \ddots & r_{d-1} \\
r_{k} & \cdots & \cdots & r_{0} & 0 & \cdots & 0 & 1
\end{array}\right) .
$$

Then

$$
\mathcal{E}_{d}=\left\{\begin{array}{l|l}
\left(r_{0}, \ldots, r_{d-1}\right) \in \mathbb{R}^{d} & \begin{array}{r}
\forall k \in\{0, \ldots, d-1\} \text { we have } \\
\operatorname{det}\left(\delta_{k}\left(r_{0}, \ldots, r_{d-1}\right)\right)>0
\end{array}
\end{array}\right\} .
$$

For small $d$ we have

$$
\begin{aligned}
& \mathcal{E}_{1}=\{x \in \mathbb{R}|| x \mid<1\} \\
& \mathcal{E}_{2}=\left\{(x, y) \in \mathbb{R}^{2}|| x|<1,| y \mid<x+1\right\} \\
& \mathcal{E}_{3}=\left\{(x, y, z) \in \mathbb{R}^{3}|| x|<1,| y-x z\left|<1-x^{2},\right| x+z \mid<y+1\right\} .
\end{aligned}
$$

We already saw that $\mathcal{E}_{2}$ is a triangle. $\mathcal{E}_{3}$ is depicted in Figure 2.1. For obtaining a characterization of $\mathcal{E}_{d}$ in the above mentioned way we have to calculate $d$ determinants, the biggest of them having dimension $2 d \times$ $2 d$. There exists another way of characterizing $\mathcal{E}_{d}$ which is based on the 
results proved by Fam and Meditch in [9]. In this paper it was shown that $\mathcal{E}_{d}$ is simply connected and that for $d \geq 2$ it is bounded by three hypersurfaces two of which are hyperplanes. Just intuitively this is obvious by the following observations.

Indeed, for $\mathbf{r} \in \mathbb{R}^{d}$ denote by $\lambda_{1}, \ldots, \lambda_{d}$ the $d$ (not necessarily distinct) roots of the characteristic polynomial $\chi_{R(\mathbf{r})}$ of $R(\mathbf{r})$. It is easy to see that $\mathbf{r} \in \mathcal{E}_{d}$ if and only if $\left|\lambda_{i}\right|<1$ for all $i \in\{1, \ldots, d\}$ and $\mathbf{r} \in \partial \mathcal{E}_{d}$ if and only if $\left|\lambda_{i}\right| \leq 1$ for all $i \in\{1, \ldots, d\}$ and equality holds for at least one index. There are three possibilities for a root to have modulus 1 . It can be $+1,-1$ or a non-real complex number with absolute value 1 . Each of these possibilities corresponds to one hypersurface. For obtaining a parametrization of these hypersurfaces define for vectors $\mathbf{r}=\left(r_{0}, \ldots, r_{p-1}\right), \mathbf{s}=\left(s_{0}, \ldots, s_{q-1}\right)$ of arbitrary dimension $p, q \in \mathbb{N}$ the binary function $\odot$ by

$$
\chi_{R(\mathbf{r} \odot \mathbf{s})}=\chi_{R(\mathbf{r})} \chi_{R(\mathbf{s})} .
$$

Obviously $\odot$ is associative, commutative and continuous in both arguments. Formally denote by () the 0-dimensional vector and define $\mathbf{r} \odot():=\mathbf{r}$ for all vectors $\mathbf{r}$. Note that this makes $\left(\bigcup_{k \in \mathbb{N}} \mathbb{R}^{k}, \odot\right)$ a commutative monoid which is isomorphic to the monoid of monic real polynomials together with multiplication. Furthermore, it is convenient to denote by $\mathcal{E}_{0}$ the set that consists only of (). For $D \subset \mathbb{R}^{p}$ and $E \subset \mathbb{R}^{q}$ let $D \odot E:=\{\mathbf{r} \odot \mathbf{s} \mid \mathbf{r} \in D, \mathbf{s} \in$ $E\}$. The following results have already been presented by Fam and Meditch in [9]. Since the authors only give sketched and somewhat heuristic proofs we prove the results in full detail here.

Lemma 2.1 ( $c f$. [9, Formulas (9) and (10)]). Let $p, q \in \mathbb{N}, p$ even. Then

(1) $\frac{\mathcal{E}_{p}}{\varepsilon_{p}} \odot \frac{\mathcal{E}_{q}}{\varepsilon_{q}}=\underline{\mathcal{E}_{p+q}}$,

(2) $\overline{\mathcal{E}_{p}} \odot \overline{\mathcal{E}_{q}}=\overline{\mathcal{E}_{p+q}}$,

(3) $\partial \mathcal{E}_{p} \odot \overline{\mathcal{E}_{q}} \cup \overline{\mathcal{E}_{p}} \odot \partial \mathcal{E}_{q}=\partial \mathcal{E}_{p+q}$.

Proof. For $p=0$ or $q=0$ the lemma is trivial. Thus suppose $p$ and $q$ are non-zero. For proving (1) suppose that $\mathbf{r}:=\left(r_{0}, \ldots, r_{p-1}\right) \in \mathcal{E}_{p}$ and $\mathbf{r}^{\prime}:=\left(r_{0}^{\prime}, \ldots, r_{q-1}^{\prime}\right) \in \mathcal{E}_{q}$. This implies that the roots of the polynomials $P(x)=\chi_{R(\mathbf{r})}(x)=x^{p}+r_{p-1} x^{p-1}+\cdots+r_{0}$ and $Q(x)=\chi_{R\left(\mathbf{r}^{\prime}\right)}(x)=$ $x^{q}+r_{q-1}^{\prime} x^{q-1}+\cdots+r_{0}^{\prime}$ have modulus smaller than 1 . Thus, by (2.3), we have $\chi_{R\left(\mathbf{r} \odot \mathbf{r}^{\prime}\right)}=P Q$ and therefore $\mathbf{r} \odot \mathbf{r}^{\prime} \in \mathcal{E}_{p+q}$. On the other hand suppose that $\mathbf{s} \in \mathcal{E}_{p+q}$. We have to show that there exist $\mathbf{r} \in \mathcal{E}_{p}$ and $\mathbf{r}^{\prime} \in \mathcal{E}_{q}$ with $\mathbf{r} \odot \mathbf{r}^{\prime}=\mathbf{s}$. We can write $\chi_{R(\mathbf{s})}$ as a product of the shape

$$
\begin{aligned}
\chi_{R(\mathbf{s})}(x)=\left(x-\lambda_{1}\right) \cdots\left(x-\lambda_{s}\right) & \left(x-\lambda_{s+1}\right) \cdots \\
& \cdots\left(x-\lambda_{s+t}\right)\left(x-\lambda_{s+t+1}\right) \cdots\left(x-\lambda_{s+2 t}\right)
\end{aligned}
$$

with $s, t \geq 0, s+2 t=p+q, \lambda_{j} \in \mathbb{R}$ for $1 \leq j \leq s, \lambda_{s+j} \in \mathbb{C} \backslash \mathbb{R}$ for $1 \leq j \leq 2 t, \lambda_{s+j}=\overline{\lambda_{s+t+j}}$ for $1 \leq j \leq t$ and $\left|\lambda_{j}\right|<1$ for $1 \leq j \leq p+q$. Let 
$J \subset\{1, \ldots, s\}$ and $J^{\prime} \subset\{1, \ldots, t\}$ such that $|J|+2\left|J^{\prime}\right|=p$. This is always possible, since $p$ is even. Let

$$
\begin{aligned}
P(x) & =x^{p}+r_{p-1} x^{p-1}+\cdots+r_{0} \\
& :=\prod_{j \in J}\left(x-\lambda_{j}\right) \prod_{j \in J^{\prime}}\left(x-\lambda_{s+j}\right)\left(x-\lambda_{s+t+j}\right), \\
Q(x) & =x^{q}+r_{q-1}^{\prime} x^{q-1}+\cdots+r_{0}^{\prime} \\
& :=\prod_{j \in\{1, \ldots, s\} \backslash J}\left(x-\lambda_{j}\right) \prod_{j \in\{1, \ldots, t\} \backslash J^{\prime}}\left(x-\lambda_{s+j}\right)\left(x-\lambda_{s+t+j}\right) .
\end{aligned}
$$

Note that $P$ and $Q$ are real polynomials. Then obviously $\mathbf{r}:=\left(r_{0}, \ldots, r_{p-1}\right)$ $\in \mathcal{E}_{p}, \mathbf{r}^{\prime}:=\left(r_{0}^{\prime}, \ldots, r_{q-1}^{\prime}\right) \in \mathcal{E}_{q}$ and $\mathbf{r} \odot \mathbf{r}^{\prime}=\mathbf{s}$ completing the proof of Assertion (1). Assertions (2) and (3) follow immediately by the continuity of $\odot$.

Note that for $p \equiv q \equiv 1 \bmod 2$ we only have $\mathcal{E}_{p} \odot \mathcal{E}_{q} \subset \mathcal{E}_{p+q}$. Lemma 2.1 can be used to obtain a parametrization of $\mathcal{E}_{p+q}$ from parametrizations of $\mathcal{E}_{p}$ and $\mathcal{E}_{q}$. We will show this by an example.

Example. It is easy to see that $\mathcal{E}_{1}=\{v \mid-1<v<1\}$ and $\mathcal{E}_{2}=$ $\{(s,(s+1) t) \mid-1<s, t<1\}$. We now want to get a parametrization of $\mathcal{E}_{3}$. From Assertion (1) of Lemma 2.1 we know that $\mathcal{E}_{3}=\mathcal{E}_{1} \odot \mathcal{E}_{2}$. By the definition of $\odot$ this shows that

$$
\mathcal{E}_{3}=\{(v s, s+v t(s+1),(s+1) t+v) \mid-1<s, t, v<1\} .
$$

This parametrization maps $(-1,1)^{3}$ onto $\mathcal{E}_{3}$. However, this mapping is not bijective since, for example, we have $(0) \odot\left(\frac{1}{6}, \frac{5}{6}\right)=\left(\frac{1}{2}\right) \odot\left(0, \frac{1}{3}\right)$. By Assertion (2) of Lemma 2.1 we easily get a parametrization of $\overline{\mathcal{E}_{3}}$ by enlarging the domain of $s, t, v$ to the closed interval $[-1,1]$. This shows the advantage of the representation (2.4) since exchanging the strict inequalities to non-strict ones in the representation of $\mathcal{E}_{3}$ in $(2.2)$ does not yield $\overline{\mathcal{E}_{3}}$ ( $c f$. [11]).

Now we turn to the boundary of $\mathcal{E}_{d}$. Assertion (3) of Lemma 2.1 already shows one way to obtain $\partial \mathcal{E}_{d}$. Now we will present an alternative representation. Let $d \geq 2$ and

$$
\begin{aligned}
E_{d}^{(1)} & :=(1) \odot \overline{\mathcal{E}_{d-1}}, \\
E_{d}^{(-1)} & :=(-1) \odot \overline{\mathcal{E}_{d-1}}, \\
E_{d}^{(\mathbb{C})} & :=\{(1, a) \mid a \in(-2,2)\} \odot \overline{\mathcal{E}_{d-2}} .
\end{aligned}
$$

Theorem 2.1 ( $c f$. [9, Section II]). For $d \geq 3$ we have

$$
\partial \mathcal{E}_{d}=E_{d}^{(1)} \cup E_{d}^{(-1)} \cup E_{d}^{(\mathbb{C})} .
$$


Proof. The proof runs along similar lines as the proof of Lemma 2.1. Since $(1),(-1) \in \partial \mathcal{E}_{1}$ and $\{(1, a) \mid a \in(-2,2)\} \in \partial \mathcal{E}_{2}$ we have $E_{d}^{(1)} \cup E_{d}^{(-1)} \cup E_{d}^{(\mathbb{C})} \subset$ $\partial \mathcal{E}_{d}$. On the other hand suppose that $\mathbf{r} \in \partial \mathcal{E}_{d}$. Then $\chi_{R(\mathbf{r})}$ has a root 1 , a root -1 or a pair of complex conjugate roots with absolute value 1 . All other roots have modulus less than or equal to 1 . Suppose $\chi_{R(\mathbf{r})}(1)=0$. Then $\chi_{R(\mathbf{r})}(x)=(x-1) \chi_{R(\mathbf{s})}(x)$ for some $\mathbf{s} \in \mathbb{R}^{d-1}$ and thus $\mathbf{r}=(-1) \odot \mathbf{s}$. Since all roots of $\chi_{R(\mathbf{r})}$ have modulus less than or equal to 1 we necessarily have $\mathbf{s} \in \overline{\mathcal{E}_{d-1}}$ showing that $\mathbf{r} \in E_{d}^{(-1)}$. Similarly it can be shown that $\mathbf{r} \in E_{d}^{(1)}$ when $\chi_{R(\mathbf{r})}$ has -1 as a root. If $\chi_{R(\mathbf{r})}$ has a pair of complex conjugate roots having modulus 1 , say $\alpha \pm i \beta,|\alpha|<1$, then $\chi_{R(\mathbf{r})}(x)$ must be divisible by $x^{2}-2 \alpha+1$. Hence $\chi_{R(\mathbf{r})}(x)=\left(x^{2}-2 \alpha+1\right) \chi_{R(\mathbf{s})}(x)$ for some $\mathbf{s} \in \mathbb{R}^{d-2}$ and we even have $\mathbf{s} \in \overline{\mathcal{E}_{d-2}}$ by the same argument as above. Thus $\mathbf{r}=(1,-2 \alpha) \odot \mathbf{s} \in E_{d}^{(\mathbb{C})}$.

Note that in view of (2.5) this shows that $E_{d}^{(1)}$ and $E_{d}^{(-1)}$ are homeomorphic images of $\overline{\mathcal{E}_{d-1}}$. It is not true that $E_{d}^{(\mathbb{C})}$ is a homeomorphic image of $\overline{\mathcal{E}_{d-2}} \times(-2,2)$ for $d \geq 4$, as injectivity is violated. This can be seen by observing that for $a, b \in(-2,2)$ with $a \neq b$ we have $\mathbf{r}:=(0, \ldots, 0,1, a) \in \partial \mathcal{E}_{d-2}$, $\mathbf{s}:=(0, \ldots, 0,1, b) \in \partial \mathcal{E}_{d-2}$ and $\mathbf{r} \odot(1, b)=\mathbf{s} \odot(1, a)$. However, it is easy to see that the interior of $E_{d}^{(\mathbb{C})}$ (subspace topology) is always a homeomorphic image of $\mathcal{E}_{d-2} \times(-2,2)$.

Theorem 2.2 (cf. $[9$, Section $\mathrm{II}]) \cdot E_{d}^{(1)}, E_{d}^{(-1)}$ and $E_{d}^{(\mathbb{C})}$ are simply connected. Moreover, $E_{d}^{(1)}$ and $E_{d}^{(-1)}$ are subsets of hyperplanes.

Proof. The simple connectivity of $E_{d}^{(1)}, E_{d}^{(-1)}$ and $E_{d}^{(\mathbb{C})}$ follows immediately from the simple connectivity of $\mathcal{E}_{d-1}$ and $\mathcal{E}_{d-2}$ and by the definition of $E_{d}^{(1)}, E_{d}^{(-1)}$ and $E_{d}^{(\mathbb{C})}$. Now $\mathbf{x}=\left(x_{0}, \ldots, x_{d-1}\right) \in E_{d}^{(1)}$ if and only if there exists a (unique) point $\mathbf{r}=\left(r_{0}, \ldots, r_{d-2}\right) \in \mathcal{E}_{d-1}$ such that

$$
\mathbf{x}=(1) \odot \mathbf{r}=\left(r_{0}, r_{0}+r_{1}, \ldots, r_{d-3}+r_{d-2}, r_{d-2}+1\right) .
$$

Thus $\left(x_{0}, \ldots, x_{d-1}\right)$ satisfies $1+\sum_{j=1}^{d}(-1)^{j} x_{d-j}=0$ showing that $E_{d}^{(1)}$ lies on a hyperplane. Similarly it can be shown that

$$
E_{d}^{(-1)} \subset\left\{\left(X_{0}, \ldots, X_{d-1}\right) \in \mathbb{R}^{d} \mid X_{0}+\cdots+X_{d-1}+1=0\right\} .
$$

Let us summarize some facts on $\mathcal{E}_{d}$. Fam and Meditch show that $\mathcal{E}_{d}$ is bounded ( $c f$. [9, Theorem 1]) and simply connected ( $c f .[9$, Lemma 1]). As mentioned in (1.2), it satisfies $\mathcal{E}_{d}=\operatorname{int}\left(\overline{\mathcal{E}_{d}}\right)$. Moreover, Theorem 2.1 yields that $\mathcal{E}_{d}$ is bounded by three hypersurfaces for $d \geq 2$. Two of these hypersurfaces are hyperplanes. If $d=2$ the third one is a hyperplane (line), 
too. This is obviously not true for $d \geq 3 . E_{d}^{(\mathbb{C})}$ is a subset of an algebraic curve of higher degree. Just for completeness we want to mention another result of Fam [8] in which the volume $V\left(\mathcal{E}_{d}\right)$ of $\mathcal{E}_{d}$ is calculated. Using the Barnes G-function $G$ we have

$$
V\left(\mathcal{E}_{d}\right)= \begin{cases}\frac{2^{2 n^{2}+n} \Gamma(n+1) G(n+1)^{4}}{G(2 n+2)} & (d=2 n), \\ \frac{2^{2 n^{2}+3 n+1} G(n+2)^{4}}{\Gamma(n+1) G(2 n+3)} & (d=2 n+1) .\end{cases}
$$

Note that for positive integers the Barnes G-function equals the superfactorials: $G(n+2)=\prod_{j=1}^{n} j$ ! for $n \in \mathbb{N}$. Moreover, observe that by [8, Formula (2.13)] we have $\lim _{d \rightarrow \infty} V\left(\mathcal{E}_{d}\right)=0$. On the other hand the diameter of $\mathcal{E}_{d}$ tends to infinity with $d$. Indeed, the vector of the coefficients of the $k$-th cyclotomic polynomial $\Phi_{k}$ belongs to the boundary of $\mathcal{E}_{\varphi(k)}$ and by a result of Emma Lehmer [13] the maximum of the absolute value of the coefficients of $\Phi_{k}$ is not bounded.

\section{First relations between the orbits of $\tau_{\mathrm{r}}$ and $\tau_{\mathrm{r} \odot \mathrm{s}}$}

In this section we will investigate the relation between the behavior of $\tau_{\mathbf{r}}$ and the behavior of $\tau_{\mathbf{r} \odot \mathbf{s}}$ for $\mathbf{r} \in \mathbb{R}^{d}$ and $\mathbf{s} \in \mathbb{Z}^{q}$ (see Theorem 3.1). We will see that it is necessary for $\mathbf{r}$ to be an element of $\mathcal{D}_{d}$ in order to have that $\mathbf{r} \odot \mathbf{s} \in \mathcal{D}_{d+q}$. Moreover, we need $\mathbf{s}$ to have integer coordinates because otherwise there exist no relations between $\tau_{\mathbf{r}}$ and $\tau_{\mathbf{r} \odot \mathbf{s}}$ as the ones described in Theorem 3.1.

This forms the basis for a finer classification of $\mathcal{D}_{d}$ which will be established in the subsequent sections and which will enable us to give necessary and sufficient conditions for $\mathbf{r}$ and $\mathbf{s}$ to ensure that $\mathbf{r} \odot \mathbf{s} \in \mathcal{D}_{d+q}$. These conditions can be checked algorithmically. Thus at the end we will be able to describe large pieces of $\mathcal{D}_{d+q} \backslash \mathcal{E}_{d+q}$.

We start with an analysis on how the maps $\tau_{\mathbf{r}}$ and $\odot$ fit together. At first we introduce a new map acting on (right) infinite sequences. For some $q \in \mathbb{N} \backslash\{0\}$ and a vector $\mathbf{s}=\left(s_{0}, \ldots, s_{q-1}\right) \in \mathbb{Z}^{q}$ define

$$
V_{\mathbf{s}}: \mathbb{Z}^{\infty} \rightarrow \mathbb{Z}^{\infty}, \quad\left(x_{n}\right)_{n \in \mathbb{N}} \mapsto\left(\sum_{k=0}^{q-1} s_{k} x_{n+k}+x_{n+q}\right)_{n \in \mathbb{N}} .
$$

It is easy to see that $V_{\mathbf{s}}$ maps each periodic sequence to a periodic sequence and each sequence that is eventually zero to a sequence that is eventually zero.

Let $\mathbf{r} \in \mathbb{R}^{d}$ and $\mathbf{x} \in \mathbb{Z}^{d}$. Successive application of $\tau_{\mathbf{r}}$ to $\mathbf{x}$ induces a sequence of integer vectors, $\left(\mathbf{x}_{n}\right)_{n \in \mathbb{N}}=\left(\tau_{\mathbf{r}}^{n}(\mathbf{x})\right)_{n \in \mathbb{N}}$, where $\mathbf{x}_{0}=\mathbf{x}$. By the definition of $\tau_{\mathbf{r}}$ after each application only one entry changes. The other entries only change their position. Denote by $\tau_{\mathbf{r}}^{*}(\mathbf{x}) \in \mathbb{Z}^{\infty}$ the sequence $\left(x_{n}\right)_{n \in \mathbb{N}}$ of the first entries of the sequence $\left(\mathbf{x}_{n}\right)_{n \in \mathbb{N}}$. It is easy to 
see that for all $k \geq 0$ we have $\left(x_{k}, \ldots, x_{k+d-1}\right)=\mathbf{x}_{k}=\tau_{\mathbf{r}}^{k}(\mathbf{x})$, in particular, $\mathbf{x}=\left(x_{0}, \ldots, x_{d-1}\right)$. We will denote a sequence $\left(x_{n}\right)_{n \in \mathbb{N}}$ with period $l$ and preperiod $k$ by $x_{0}, \ldots, x_{k-1},\left(x_{k}, \ldots, x_{l+k-1}\right)^{\infty}$. If there is an index $k$ such that $x_{n}=0$ for $n \geq k$ we will therefore write $x_{0}, \ldots, x_{k-1},(0)^{\infty}$.

The behavior of $\tau_{\mathbf{r}}$ can be described completely by the behavior of $\tau_{\mathbf{r} \odot \mathbf{s}}$ if $\mathbf{s} \in \mathbb{Z}^{q}$. This is made precise in the following theorem. If $\mathbf{s} \in \partial \mathcal{E}_{q}$ then also $\mathbf{r} \odot \mathbf{s} \in \partial \mathcal{E}_{d+q}$. Thus this result will enable us to describe parts of $\mathcal{D}_{d+q} \backslash \mathcal{E}_{d+q} \subset \partial \mathcal{E}_{d+q}$.

Theorem 3.1. Let $p, q \geq 1$ be integers, $\mathbf{r} \in \mathbb{R}^{p}$ and $\mathbf{s} \in \mathbb{Z}^{q}$. Then

$$
V_{\mathbf{s}} \circ \tau_{\mathbf{r} \odot \mathbf{s}}^{*}\left(\mathbb{Z}^{p+q}\right)=\tau_{\mathbf{r}}^{*}\left(\mathbb{Z}^{p}\right) .
$$

Proof. Suppose $\mathbf{r}=\left(r_{0}, \ldots, r_{p-1}\right)$ and $\mathbf{s}=\left(s_{0}, \ldots, s_{q-1}\right)$. Set

$$
U=\left(\begin{array}{cccccccc}
s_{0} & s_{1} & \cdots & s_{q-1} & 1 & 0 & \cdots & 0 \\
0 & s_{0} & & & \ddots & \ddots & \ddots & \vdots \\
\vdots & \ddots & \ddots & & & \ddots & \ddots & 0 \\
0 & \cdots & 0 & s_{0} & \cdots & \cdots & s_{q-1} & 1
\end{array}\right) \in \mathbb{Z}^{p \times p+q} .
$$

Note that $U$ has maximal rank $p$ and $U \mathbb{Z}^{p+q}=\mathbb{Z}^{p}$. Thus the theorem is proved if we show that for all $\mathbf{x} \in \mathbb{Z}^{p+q}$ we have

$$
V_{\mathbf{s}} \circ \tau_{\mathbf{r} \odot \mathbf{s}}^{*}(\mathbf{x})=\tau_{\mathbf{r}}^{*}(U \mathbf{x}) .
$$

Suppose $\left(x_{k}\right)_{k \in \mathbb{N}}=\tau_{\mathbf{r} \odot \mathbf{s}}^{*}(\mathbf{x})$ and $\left(y_{k}\right)_{k \in \mathbb{N}}=\tau_{\mathbf{r}}^{*}(U \mathbf{x})$. We have to show that

$$
y_{n}=s_{0} x_{n}+\cdots+s_{q-1} x_{n+q-1}+x_{n+q}
$$

holds for all $n \geq 0$. We do this by induction on $n$. For $0 \leq n \leq p-1$ this is true by the definition of $U$. Now assume that (3.2) is true for each $k \in\{0, \ldots, n\}$ with $n \geq p-1$. By the definition of $\left(y_{k}\right)_{k \in \mathbb{N}}$ we have

$$
y_{n+1}=-\left\lfloor\sum_{j=0}^{p-1} r_{j} y_{n+1-p+j}\right\rfloor \text {. }
$$

By the assumption on the induction this gives

$$
\begin{aligned}
y_{n+1}=- & \left.\sum_{j=0}^{p-1} r_{j}\left(s_{0}, \ldots, s_{q-1}, 1\right) \cdot\left(x_{n+1-p+j}, \ldots, x_{n+1+q-p+j}\right)\right\rfloor \\
=- & \left\lfloor\sum_{j=0}^{p-1} r_{j}\left(s_{0}, \ldots, s_{q-1}, 1\right) \cdot\left(x_{n+1-p+j}, \ldots, x_{n+1+q-p+j}\right)\right. \\
& \left.+\sum_{i=0}^{q-1} s_{i} x_{n+1+i}\right\rfloor+\sum_{i=0}^{q-1} s_{i} x_{n+1+i},
\end{aligned}
$$


where we used the fact that $\mathbf{s}$ is an integer vector. Now write the argument of the last floor function as scalar product of two $p+d$ dimensional vectors. It is easy to see that this gives

$$
\begin{aligned}
& =\left\lfloor\left(r_{0}\left(\begin{array}{c}
s_{0} \\
\vdots \\
s_{q-1} \\
1 \\
0 \\
\vdots \\
\vdots \\
0
\end{array}\right)+\cdots+r_{p-1}\left(\begin{array}{c}
0 \\
\vdots \\
0 \\
s_{0} \\
s_{1} \\
\vdots \\
s_{q-1} \\
1
\end{array}\right)+\left(\begin{array}{c}
0 \\
\vdots \\
\vdots \\
0 \\
s_{0} \\
s_{1} \\
\vdots \\
s_{q-1}
\end{array}\right)\right)\right. \\
=- & \left.(\mathbf{s} \odot \mathbf{r})\left(\begin{array}{c}
x_{n+1-p} \\
\vdots \\
x_{n+q}
\end{array}\right)\right\rfloor=x_{n+q+1}
\end{aligned}
$$

where the last equality follows from the definition of $\left(x_{k}\right)_{k \in \mathbb{N}}$. Thus we arrive at

$$
y_{n+1}=s_{0} x_{n+1}+\cdots+s_{q-1} x_{n+q}+x_{n+q+1}
$$

which shows that (3.2) holds for $n+1$. Hence, in view of (3.1) the theorem is proved.

We give an example for illustrating the statement of the theorem.

Example. Let $\mathbf{r}=\left(\frac{11}{12}, \frac{9}{5}\right)$ and $\mathbf{s}=(1)$. Theorem 3.1 says that for each $\mathbf{y} \in \mathbb{Z}^{2}$ there exists $\mathbf{x} \in \mathbb{Z}^{3}$ such that $\tau_{\mathbf{r}}^{*}(\mathbf{y})=V_{\mathbf{s}} \circ \tau_{\mathbf{r} \odot \mathbf{s}}^{*}(\mathbf{x})$, i.e., the behavior of $\tau_{\mathbf{r}}$ is completely described by the behavior of $\tau_{\mathbf{r}} \odot \mathbf{s}$. For instance, suppose $\mathbf{y}:=(5,-3)$. By $(3.1)$ we have to choose $\mathbf{x}$ such that $U \mathbf{x}=\mathbf{y}$ with

$$
U=\left(\begin{array}{lll}
1 & 1 & 0 \\
0 & 1 & 1
\end{array}\right)
$$

Hence, we can choose $\mathbf{x}:=(4,1,-4)$. We have $\mathbf{r} \odot \mathbf{s}=\left(\frac{11}{12}, \frac{163}{60}, \frac{14}{5}\right)$ and

$$
\tau_{\mathbf{r} \odot \mathbf{s}}^{*}(\mathbf{x})=4,1,-4,(5,-4,2,1,-4,7,-9,10,-9,7,-4,1,2,-4)^{\infty} .
$$

In our case the map $V_{\mathbf{s}}$ adds two consecutive entries of a sequence. We thus have

$$
V_{\mathbf{s}} \circ \tau_{\mathbf{r} \odot \mathbf{s}}^{*}(\mathbf{x})=5,-3,(1,1,-2,3,-3,3,-2)^{\infty}=\tau_{\mathbf{r}}^{*}(\mathbf{y}) .
$$

From Theorem 3.1 we can deduce several assertions. Firstly by choosing $\mathbf{s}=(0)$ we obtain that

$$
\begin{aligned}
\left(r_{0}, \ldots, r_{d-1}\right) \in \mathcal{D}_{d} & \Longleftrightarrow\left(0, r_{0}, \ldots, r_{d-1}\right) \in \mathcal{D}_{d+1} \\
\left(r_{0}, \ldots, r_{d-1}\right) \in \mathcal{D}_{d}^{(0)} & \Longleftrightarrow\left(0, r_{0}, \ldots, r_{d-1}\right) \in \mathcal{D}_{d+1}^{(0)}
\end{aligned}
$$


which is contained in [1].

More generally we have the following result.

Corollary 3.1. Let $\mathbf{r} \in \mathbb{R}^{d}$ and $\mathbf{s} \in \overline{\mathcal{E}_{q}} \cap \mathbb{Z}^{q}$.

- If $\mathbf{r} \odot \mathbf{s} \in \mathcal{D}_{d+q}$ then $\mathbf{r} \in \mathcal{D}_{d}$.

- If $\mathbf{r} \odot \mathbf{s} \in \mathcal{D}_{d+q}^{(0)}$ then $\mathbf{r} \in \mathcal{D}_{d}^{(0)}$.

Proof. This can be seen immediately by Theorem 3.1 since $V_{\mathbf{s}}$ preserves the periodicity of a sequence as well as the fact that a sequence is eventually zero.

Note that $\mathbf{s} \in \overline{\mathcal{E}_{q}} \cap \mathbb{Z}^{q}$ holds if and only if $\chi_{R(\mathbf{s})}$ is the product of cyclotomic polynomials.

We restricted ourselves to integer vectors $\mathbf{s} \in \overline{\mathcal{E}_{q}}$ since for $\mathbf{s} \notin \overline{\mathcal{E}_{q}}$ we had $\mathbf{r} \odot \mathbf{s} \notin \overline{\mathcal{E}_{d+q}}$ and thus $\mathbf{r} \odot \mathbf{s} \in \mathcal{D}_{d+q}\left(\mathbf{r} \odot \mathbf{s} \in \mathcal{D}_{d+q}^{(0)}\right.$, respectively) cannot hold in view of Lemma $2.1(3)$. Observe that $\mathbf{s} \in \overline{\mathcal{E}_{q}} \cap \mathbb{Z}^{q}$ implies $\mathbf{s} \in \partial \mathcal{E}_{q}$ whenever $\mathbf{s} \neq \mathbf{0}$ and thus $\mathbf{r} \odot \mathbf{s} \in \partial \mathcal{E}_{d+q}$. Thus the above corollary yields first results concerning the relation between $\mathcal{D}_{d+q} \backslash \mathcal{E}_{d+q}$ and $\mathcal{D}_{d}\left(\mathcal{D}_{d+q}^{(0)} \backslash \mathcal{E}_{d+q}\right.$ and $\mathcal{D}_{d}^{(0)}$, respectively). It should be mentioned that the second part of Corollary 3.1 is of interest only if there exists $d \in \mathbb{N}$ with $\mathcal{D}_{d}^{(0)} \backslash \mathcal{E}_{d} \neq \emptyset$. Up to now no such $d$ is known. As mentioned in the introduction, for $d=2$ it is shown in $[4$, Section 2] that the intersection is empty

Note that the converse of Corollary 3.1 does not hold in general. It is shown in [2] that $\left(1, \frac{1+\sqrt{5}}{2}\right) \in \mathcal{D}_{2}$. But the already mentioned analysis of Kirschenhofer et al. [12] shows that $\left(1, \frac{3+\sqrt{5}}{2}, \frac{3+\sqrt{5}}{2}\right)=(1) \odot\left(1, \frac{1+\sqrt{5}}{2}\right) \in$ $\partial \mathcal{E}_{3} \backslash \mathcal{D}_{3}$. In the following we will develop a converse of Corollary 3.1 that gives necessary and sufficient conditions for $\mathbf{s}$ and the behavior of $\tau_{\mathbf{r}}$ to ensure that $\mathbf{r} \odot \mathbf{s} \in \mathcal{D}_{d+p}$.

\section{Some classes of cycles of $\tau_{\mathrm{r}}$}

This section is devoted to solving the problem of finding sufficient conditions for $\mathbf{r} \in \mathcal{D}_{d}$ such that the converse of Corollary 3.1 holds. To this matter we will define sets characterized by cycles of $\tau_{\mathbf{r}}$ with certain properties. First we need some additional definitions and notations. Define the following equivalence relation for finite sequences.

$$
\begin{aligned}
\left(x_{0}, \ldots, x_{l-1}\right) & \cong\left(y_{0}, \ldots, y_{m-1}\right) \Longleftrightarrow \\
\exists k & \in \mathbb{N}: x_{0}=y_{k}, x_{1}=y_{k+1}, \ldots, x_{l \mathrm{~cm}(l, m)-1}=y_{k+\operatorname{lcm}(l, m)-1}
\end{aligned}
$$

where the indices of $x$ and $y$ are taken modulo $l$ and $m$, respectively. It is easy to see that $\cong$ is an equivalence relation. Let $\mathbb{Z}^{*}$ the set of all finite 
integer sequences. We will denote the elements of $\mathbb{Z}^{*} / \cong$ by representatives of minimal length inside angle brackets. For example, we have

$$
(2,3,1,2,3,1) \cong(3,1,2,3,1,2,3,1,2) \cong(1,2,3)
$$

and the corresponding equivalence class is $\langle 1,2,3\rangle(=\langle 3,1,2\rangle=\langle 2,3,1\rangle)$. Furthermore, let

$$
\pi: \mathbb{Z}^{*} \rightarrow \mathbb{Z}^{*} / \cong
$$

be the canonical epimorphism and

$$
l: \mathbb{Z}^{*} \rightarrow \mathbb{N}
$$

the length of the representative. Note that the length is well defined. In the example from above we have $l(\langle 1,2,3\rangle)=3$.

For $\mathbf{r} \in \mathcal{D}_{d}$ the sequences $\tau_{\mathbf{r}}^{*}(\mathbf{x})$ end up periodically for all choices of $\mathbf{x} \in \mathbb{Z}^{d}$. This motivates the following definition.

Definition. For $\mathbf{r} \in \mathcal{D}_{d}$ we call a point $\mathbf{x} \in \mathbb{Z}^{d}$ purely periodic (with respect to $\left.\tau_{\mathbf{r}}\right)$ if there exists an $l \in \mathbb{N}$ such that $\tau_{\mathbf{r}}^{l}(\mathbf{x})=\mathbf{x}$. Moreover let

$$
C(\mathbf{r}):=\pi\left\{b \in \mathbb{Z}^{*} \mid \exists \mathbf{x} \in \mathbb{Z}^{d}: \tau_{\mathbf{r}}^{*}(\mathbf{x})=b^{\infty}\right\} \subset \mathbb{Z}^{*} / \cong
$$

be the set of all equivalence classes of cycles of $\tau_{\mathbf{r}}$. For $p \in \mathbb{N} \backslash\{0\}$, define the function

$$
\begin{aligned}
S_{p}: \mathbb{Z}^{*} / \cong & \rightarrow\{0,1\} \\
\mathcal{B}=\left\langle x_{0}, \ldots, x_{l(\mathcal{B})-1}\right\rangle & \mapsto \begin{cases}0 & \text { for } p \nmid l(\mathcal{B}) \text { or } \sum_{j=0}^{l(\mathcal{B})-1} \xi_{p}^{j} x_{j}=0 \\
1 & \text { otherwise, }\end{cases}
\end{aligned}
$$

where $\xi_{p}$ denotes a primitive $p$-th root of unity.

Note that the function $S_{p}$ is well defined since it is independent of the chosen representative of $\mathcal{B}$. We can define the set $\mathcal{D}_{d}^{(0)}$ in terms of $C(\mathbf{r})$ by

$$
\mathcal{D}_{d}^{(0)}=\left\{\mathbf{r} \in \mathcal{D}_{d} \mid C(\mathbf{r})=\{\langle 0\rangle\}\right\} .
$$

We will now make a finer classification of the elements of $\mathcal{D}_{d}$. For $p \in \mathbb{N} \backslash\{0\}$ let

$$
\mathcal{D}_{d}^{(p)}:=\left\{\mathbf{r} \in \mathcal{D}_{d} \mid \forall \mathcal{B} \in C(\mathbf{r}): S_{p}(\mathcal{B})=0\right\}
$$

We obviously have $\mathcal{D}_{d}^{(0)} \subseteq \mathcal{D}_{d}^{(p)}$ for each $p \in \mathbb{N}$. According to the following lemma, the set $\mathcal{D}_{d}^{(1)}$ coincides with $\mathcal{D}_{d}^{(0)}$ apart from a set of measure 0 .

Lemma 4.1. Let $\mathbf{r}=\left(r_{0}, \ldots, r_{d-1}\right) \in \mathcal{D}_{d}^{(1)} \backslash \mathcal{D}_{d}^{(0)}$. Then $\mathbf{r} \in \partial \mathcal{E}_{d}$. Moreover, $\chi_{R(\mathbf{r})}$ has a cyclotomic polynomial $\Phi_{k}, k>1$, as divisor. 
Proof. As $\mathbf{r} \in \mathcal{D}_{d}^{(1)} \backslash \mathcal{D}_{d}^{(0)}$ there exists a cycle $\mathcal{B} \neq\langle 0\rangle$ with $S_{1}(\mathcal{B})=0$. Suppose $\mathcal{B}=\left\langle x_{0}, \ldots, x_{l-1}\right\rangle$. By the definition of cycles and the behavior of $\tau_{\mathbf{r}}$ we know that $r_{0}, \ldots, r_{d-1}$ must satisfy the $l(\mathcal{B})$ inequalities

$$
0 \leq r_{0} x_{j}+\cdots+r_{d-1} x_{j+d-1}+x_{j+d}<1 \quad(0 \leq j \leq l(\mathcal{B})-1)
$$

where here (and throughout the remaining part of this proof) the indices of the $x_{i}$ have to be taken modulo $l(\mathcal{B})$. By the definition of $\mathcal{D}_{d}^{(1)}$ we have

$$
\sum_{j=0}^{l(\mathcal{B})-1} x_{j}=0 .
$$

By adding any $l(\mathcal{B})-1$ of the above inequalities we thus obtain another set of $l(\mathcal{B})$ inequalities, namely

$$
0 \leq-r_{0} x_{j}-\cdots-r_{d-1} x_{j+d-1}-x_{j+d}<l(\mathcal{B})-1 \quad(0 \leq j \leq l(\mathcal{B})-1) .
$$

Combining (4.2) and (4.4) we get

$$
0=r_{0} x_{j}+\cdots+r_{d-1} x_{j+d-1}+x_{j+d}
$$

for all $j \in\{0, \ldots, l(\mathcal{B})-1\}$. By multiplying these equations with integers we see that

$$
\left\{\left\langle k x_{0}, \ldots, k x_{l-1}\right\rangle \mid k \in \mathbb{Z}\right\} \subset C(\mathbf{r}) .
$$

Since $\mathcal{B} \neq\langle 0\rangle$ we have that $C(\mathbf{r})$ has infinitely many elements. By $[1$, Lemma 4.2] this implies that $\mathbf{r} \in \partial \mathcal{E}_{d}$.

To prove the second assertion observe that the recurrence relations (4.3) and (4.5) for the sequence $\left(x_{j}\right)_{j \in \mathbb{N}}$ imply that the ordinary generating function $F(z):=\sum_{j \geq 0} x_{j} z^{j}$ of this sequence fulfills

$$
F(z)=\frac{P_{0}(z)}{1+z+\cdots+z^{\ell-1}}=\frac{Q_{0}(z)}{z^{d} \chi_{R(\mathbf{r})}(1 / z)},
$$

where $P_{0}, Q_{0} \in \mathbb{Z}[z]$ with $\operatorname{deg} P_{0}<\ell-1$ and $\operatorname{deg} Q_{0}<d$. The second assertion now follows.

\section{Main results}

We now come to the main results of the present paper. They show that large parts of $\mathcal{D}_{d} \backslash \mathcal{E}_{d}$ can be described by the sets $\mathcal{D}_{e}^{(p)}$ for $e<d$. In particular, we are able to describe $\mathcal{D}_{d} \cap E_{d}^{(1)}$ and $\mathcal{D}_{d} \cap E_{d}^{(-1)}$ completely in terms of these sets. In the next section we will see that the sets $\mathcal{D}_{e}^{(p)}$ can be characterized algorithmically.

Our main result is the following. As before, $\Phi_{j}$ denotes the $j$ th cyclotomic polynomial. 
Theorem 5.1. Let $d, q \geq 1, \mathbf{r} \in \mathbb{R}^{d}$ and $\mathbf{s}=\left(s_{0}, \ldots, s_{q-1}\right) \in \mathbb{Z}^{q}$ such that $s_{0} \neq 0$. Then $\mathbf{r} \odot \mathbf{s} \in \mathcal{D}_{d+q}$ if and only if the following conditions are satisfied:

(i) $\chi_{R(\mathbf{s})}=\Phi_{\alpha_{1}} \Phi_{\alpha_{2}} \cdots \Phi_{\alpha_{b}}$ for pairwise disjoint non-negative integers $\alpha_{1}, \ldots, \alpha_{b}$

(ii) $\mathbf{r} \in \bigcap_{j=1}^{b} \mathcal{D}_{d}^{\left(\alpha_{j}\right)}$

Before we give the proof we list some propositions which contain special cases of Theorem 5.1. These propositions give characterizations of the behaviour of $\tau_{\mathbf{r}}$ on some parts of $\partial \mathcal{E}_{d}$. As already mentioned in Theorem 2.1 the boundary $\partial \mathcal{E}_{d}$ is the union of the three hypersurfaces $E_{d}^{(-1)}, E_{d}^{(1)}$ and $E_{d}^{(\mathbb{C})}$. The intersection of $\mathcal{D}_{d}$ with the hyperplanes $E_{d}^{(-1)}$ and $E_{d}^{(1)}$ is completely determined by the structure of $\mathcal{D}_{d-1}^{(1)}$ and $\mathcal{D}_{d-1}^{(2)}$, respectively.

Proposition 5.1. For $d \geq 2$ we have $\mathcal{D}_{d} \cap E_{d}^{(-1)}=(-1) \odot \mathcal{D}_{d-1}^{(1)}$.

Note that by Proposition 5.1 and Lemma 4.1 we also have

$$
\mathcal{D}_{d} \cap \operatorname{int}\left(E_{d}^{(-1)}\right)=(-1) \odot\left(\mathcal{E}_{d-1} \cap \mathcal{D}_{d-1}^{(0)}\right)
$$

(the interior on the left hand side is taken with respect to the subspace topology). At the end of the present section we give a short proof of Proposition 5.1. Indeed, we show how it follows from Theorem 5.1. In the same way as Proposition 5.1 one can show that the following results are special cases of Theorem 5.1.

Proposition 5.2. For $d \geq 2$ we have $\mathcal{D}_{d} \cap E_{d}^{(1)}=(1) \odot \mathcal{D}_{d-1}^{(2)}$.

The situation for $E_{d}^{(\mathbb{C})}$ is more complicated. Here Theorem 5.1 yields only partial results. Observe that the sets on the left hand sides in Propositions $5.3-5.5$ are proper subsets of $E_{d}^{(\mathbb{C})}$.

Proposition 5.3. For $d \geq 3$ we have $\mathcal{D}_{d} \cap(1,0) \odot \overline{\mathcal{E}_{d-2}}=(1,0) \odot \mathcal{D}_{d-2}^{(4)}$.

Proposition 5.4. For $d \geq 3$ we have $\mathcal{D}_{d} \cap(1,1) \odot \overline{\mathcal{E}_{d-2}}=(1,1) \odot \mathcal{D}_{d-2}^{(3)}$.

Proposition 5.5. For $d \geq 3$ we have $\mathcal{D}_{d} \cap(1,-1) \odot \overline{\mathcal{E}_{d-2}}=(1,-1) \odot \mathcal{D}_{d-2}^{(6)}$.

Note that for $d \geq 4$ more results of this type follow from Theorem 5.1.

In order to prove Theorem 5.1 we need the following simple auxiliary lemma, whose proof is incorporated for the sake of completeness.

Lemma 5.1. Let $\left(x_{n}\right)_{n \geq 0}$ be a sequence with

$$
x_{n}=\sum_{i=1}^{L} A_{i}(n) \lambda_{i}^{n}
$$


where $A_{i}(n)=\sum_{j=0}^{m_{i}} A_{i}^{(j)} n^{j}$ are polynomials with coefficients $A_{i}^{(j)} \in \mathbb{C}$ and $\lambda_{1}, \ldots, \lambda_{L}$ are pairwise disjoint roots of unity. If $A_{i}^{(j)} \neq 0$ for some pair $(i, j)$ with $j \geq 1$ then $x_{n}$ is not bounded.

Proof. Let $q \geq 1$ be the largest integer such that there is a pair $(i, q)$ with $A_{i}^{(q)} \neq 0$. Then, after a possible rearrangement of the indices of the $\lambda_{i}$, there is a positive integer $K \leq L$ such that

$$
\left|x_{n}\right|=n^{q}\left|\sum_{i=1}^{K} A_{i}^{(q)} \lambda_{i}^{n}\right|+O\left(n^{q-1}\right) .
$$

If we can show that $B(n):=\sum_{i=1}^{K} A_{i}^{(q)} \lambda_{i}^{n}$ is bounded away from zero for infinitely many $n$, we are done. Since the $\lambda_{i}$ are roots of unity $B(n)$ is periodic in $n$ and it suffices to prove that $B(n) \neq 0$ for at least one $n$. Suppose this is false. Then

$$
\sum_{i=1}^{K} A_{i}^{(q)} \lambda_{i}^{n+k}=0 \quad(0 \leq k<K) .
$$

However, as the determinant of this system is a multiple of a Vandermonde determinant, this would imply that $A_{i}^{(q)}=0$ for all $i$, a contradiction to our assumption.

Proof of Theorem 5.1. We start with showing the sufficiency of the two conditions. Suppose that (i) and (ii) are satisfied. Let $\mathbf{x} \in \mathbb{Z}^{d+q}$ and $\left(x_{n}\right)_{n \in \mathbb{N}}:=$ $\tau_{\mathbf{s} \odot \mathbf{r}}^{*}(\mathbf{x})$. We have to show that $\left(x_{n}\right)_{n \in \mathbb{N}}$ ends up periodically. For convenience set $s_{q}:=1$. By $(3.1)$ we know that

$$
\left(y_{n}\right)_{n \in \mathbb{N}}:=\tau_{\mathbf{r}}^{*}(\mathbf{y})=V_{\mathbf{s}}\left(\left(x_{n}\right)_{n \in \mathbb{N}}\right)
$$

holds with

$$
\mathbf{y}:=\left(\sum_{i=0}^{q} s_{i} x_{i}, \sum_{i=0}^{q} s_{i} x_{i+1} \ldots, \sum_{i=0}^{q} s_{i} x_{i+d-1}\right) .
$$

As $q \geq 1$ we have $b \geq 1$ and thus $\mathbf{r} \in \mathcal{D}_{d}^{\left(\alpha_{1}\right)}$ and a fortiori $\mathbf{r} \in \mathcal{D}_{d}$. Hence, there exists an index $n_{0}$ and a (minimal) positive integer $l$ such that $y_{n}=y_{n+l}$ for $n \geq n_{0}$. We therefore have $\left\langle y_{n_{0}}, \ldots, y_{n_{0}+l-1}\right\rangle \in C(\mathbf{r})$.

The sequence $\left(x_{n}\right)_{n \geq n_{0}}$ satisfies the recurrence relation

$$
\sum_{i=0}^{q} s_{i} x_{n_{0}+k+i}=y_{n_{0}+k}=y_{n_{0}+k+l}=\sum_{i=0}^{q} s_{i} x_{n_{0}+k+l+i}
$$

for $k \geq 0$. Its characteristic equation is

$$
\left(t^{l}-1\right) \chi_{R(\mathbf{s})}(t)=0 .
$$

Denote its roots by $\lambda_{1}, \ldots, \lambda_{g}$ and suppose that they are arranged in a way that $\lambda_{1}, \ldots, \lambda_{w}$ are roots of $t^{l}-1$ only, $\lambda_{w+1}, \ldots, \lambda_{l}$ are roots of $t^{l}-1$ and 
of $\chi_{R(\mathbf{s})}(t)$, and $\lambda_{l+1}, \ldots, \lambda_{g}$ are roots of $\chi_{R(\mathbf{s})}(t)$ only. As $t^{l}-1$ as well as $\chi_{R(\mathbf{s})}(t)$ have only simple roots by the pairwise disjointness assertion in (i) we conclude $\lambda_{w+1}, \ldots, \lambda_{l}$ have multiplicity two while all the other roots have multiplicity one. Thus

$$
x_{n_{0}+k}=\sum_{j=1}^{g} A_{j}^{(0)} \lambda_{j}^{k}+\sum_{j=w+1}^{l} A_{j}^{(1)} k \lambda_{j}^{k}
$$

for $l+q$ complex constants $A_{j}^{(\nu)}$. The sequence $\left(x_{n}\right)_{n \geq n_{0}}$ is periodic if and only if $A_{j}^{(1)}=0$ for all $j \in\{w+1, \ldots, l\}$. If $x^{l}-1$ and $\chi_{R(\mathbf{s})}$ have no common roots (i.e., if $\left.\alpha_{1} \nmid l, \ldots, \alpha_{b} \nmid l\right)$ then all roots are simple and the second sum in (5.3) is empty. Thus $\left(x_{n}\right)_{n \in \mathbb{N}}$ ends up periodically in this case and we are done. Suppose in the sequel that $x^{l}-1$ and $\chi_{R(\mathbf{s})}$ have common roots, i.e., that $w<l$.

In order to calculate the constants $A_{j}^{(\nu)}$ we use (5.2) to obtain

$$
\begin{aligned}
y_{n_{0}+k} & =\sum_{j=1}^{g} A_{j}^{(0)} \sum_{h=0}^{q} s_{h} \lambda_{j}^{k+h}+\sum_{j=w+1}^{l} A_{j}^{(1)} \sum_{h=0}^{q} s_{h}(k+h) \lambda_{j}^{k+h} \\
& =\sum_{j=1}^{g} A_{j}^{(0)} \lambda_{j}^{k} \chi_{R(\mathbf{s})}\left(\lambda_{j}\right)+\sum_{j=w+1}^{l} A_{j}^{(1)}\left(k \lambda_{j}^{k} \chi_{R(\mathbf{s})}\left(\lambda_{j}\right)+\lambda_{j}^{k+1} \chi_{R(\mathbf{s})}^{\prime}\left(\lambda_{j}\right)\right) \\
& =\sum_{j=1}^{w} A_{j}^{(0)} \lambda_{j}^{k} \chi_{R(\mathbf{s})}\left(\lambda_{j}\right)+\sum_{j=w+1}^{l} A_{j}^{(1)} \lambda_{j}^{k+1} \chi_{R(\mathbf{s})}^{\prime}\left(\lambda_{j}\right) .
\end{aligned}
$$

The latter equality holds because $\chi_{R(\mathbf{s})}\left(\lambda_{j}\right)=0$ for $j>w$. Taking $k \in$ $\{0, \ldots, l-1\}$ this is a system of $l$ linear equalities for the $l$ constants $A_{1}^{(0)}, \ldots, A_{w}^{(0)}, A_{w+1}^{(1)}, \ldots, A_{l}^{(1)}$. We will show that condition (ii) implies that $A_{j}^{(1)}=0$ for each $j \in\{w+1, \ldots, l\}$. This will yield ultimate periodicity of $\left(x_{n}\right)_{n \in \mathbb{N}}$ in view of $(5.3)$.

The linear system in (5.4) can be rewritten as

$$
\left(y_{n_{0}}, \ldots, y_{n_{0}+l-1}\right)^{T}=G\left(A_{1}^{(0)}, \ldots, A_{w}^{(0)}, A_{w+1}^{(1)}, \ldots, A_{l}^{(1)}\right)^{T}
$$

with

$$
G=\left(\begin{array}{cccccc}
\chi_{R(\mathbf{s})}\left(\lambda_{1}\right) & \cdots & \chi_{R(\mathbf{s})}\left(\lambda_{w}\right) & \chi_{R(\mathbf{s})}^{\prime}\left(\lambda_{w+1}\right) \lambda_{w+1} & \cdots & \chi_{R(\mathbf{s})}^{\prime}\left(\lambda_{l}\right) \lambda_{l} \\
\chi_{R(\mathbf{s})}\left(\lambda_{1}\right) \lambda_{1} & \cdots & \chi_{R(\mathbf{s})}\left(\lambda_{w}\right) \lambda_{w} & \chi_{R(\mathbf{s})}^{\prime}\left(\lambda_{w+1}\right) \lambda_{w+1}^{2} & \cdots & \chi_{R(\mathbf{s})}^{\prime}\left(\lambda_{l}\right) \lambda_{l}^{2} \\
\vdots & & \vdots & \vdots & & \vdots \\
\chi_{R(\mathbf{s})}\left(\lambda_{1}\right) \lambda_{1}^{l-1} & \cdots & \chi_{R(\mathbf{s})}\left(\lambda_{w}\right) \lambda_{w}^{l-1} & \chi_{R(\mathbf{s})}^{\prime}\left(\lambda_{w+1}\right) \lambda_{w+1}^{l} & \cdots & \chi_{R(\mathbf{s})}^{\prime}\left(\lambda_{l}\right) \lambda_{l}^{l}
\end{array}\right) .
$$

Denote by $G_{j}$ the matrix that is obtained by exchanging the $j$ th column of $G$ by the vector $\left(y_{n_{0}}, \ldots, y_{n_{0}+l-1}\right)^{T}$. Then, by Cramer's Rule, we have

$$
A_{j}^{(1)}=\frac{\operatorname{det} G_{j}}{\operatorname{det} G}
$$


One has $\operatorname{det} G \neq 0$ since it is a non-zero multiple of the determinant of a Vandermonde matrix. We have to show that $\operatorname{det} G_{j}=0$. We have

$$
\operatorname{det} G_{j}=\prod_{k=1}^{w} \chi_{R(\mathbf{s})}\left(\lambda_{k}\right)^{l} \prod_{\substack{k=w+1 \\ k \neq j}}^{l}\left(\lambda_{k} \chi_{R(\mathbf{s})}^{\prime}\left(\lambda_{k}\right)\right)^{l} D_{j},
$$

where

$$
D_{j}:=\operatorname{det}\left(\begin{array}{ccccccc}
1 & \cdots & 1 & y_{n_{0}} & 1 & \cdots & 1 \\
\lambda_{1} & \cdots & \lambda_{j-1} & y_{n_{0}+1} & \lambda_{j+1} & \cdots & \lambda_{l} \\
\vdots & & \vdots & \vdots & & \vdots & \\
\lambda_{1}^{l-1} & \cdots & \lambda_{j-1}^{l-1} & y_{n_{0}+l-1} & \lambda_{j+1}^{l-1} & \cdots & \lambda_{l}^{l-1}
\end{array}\right) .
$$

As the products in (5.6) are obviously nonzero we have to deal with the determinant $D_{j}$. In order to calculate this determinant add the ${\overline{\lambda_{j}}}^{k+1}$ multiple of the $k$ th row to the last row for each $k \in\{1, \ldots, l-1\}$. This does not change the determinant which now can be written as

$$
D_{j}=\operatorname{det}\left(\begin{array}{ccccccc}
1 & \cdots & 1 & y_{n_{0}} & 1 & \cdots & 1 \\
\lambda_{1} & \cdots & \lambda_{j-1} & y_{n_{0}+1} & \lambda_{j+1} & \cdots & \lambda_{l} \\
\vdots & & \vdots & \vdots & & \vdots & \\
\lambda_{1}^{l-2} & \cdots & \lambda_{j-1}^{l-2} & y_{n_{0}+k+l-2} & \lambda_{j+1}^{l-2} & \cdots & \lambda_{l}^{l-2} \\
0 & \cdots & 0 & \sum_{k=0}^{l-1} \bar{\lambda}_{j}^{k+1} y_{n_{0}+k} & 0 & \cdots & 0
\end{array}\right) .
$$

We shall now prove that $\sum_{k=0}^{l-1} \bar{\lambda}_{j}^{k} y_{n_{0}+k}=0$. This implies that thus the last determinants $D_{j}, \operatorname{det} G_{j}$ and therefore $A_{j}^{(1)}$ as well are equal to zero for each $j \in\{w+1, \ldots, l\}$. Indeed, as $\lambda_{j}$ is a root of $x^{l}-1$ and $\chi_{R(\mathbf{s})}$, in view of condition (i) there exists a $p \in\{1, \ldots, b\}$ with $\alpha_{p} \mid l$ and thus $\lambda_{j}$ and, hence, $\overline{\lambda_{j}}$ as well are primitive $\alpha_{p}$ th roots of unity. As $\alpha_{p} \mid l$ and $\left\langle y_{n_{0}}, \ldots, y_{n_{0}+l-1}\right\rangle \in C(\mathbf{r})$ we have

$$
S_{\alpha_{p}}\left(\left\langle y_{n_{0}}, \ldots, y_{n_{0}+l-1}\right\rangle\right)=\sum_{k=0}^{l-1} \xi_{\alpha_{p}}^{k} y_{n_{0}+k}=0
$$

for each $\alpha_{p}$ th root of unity $\xi_{\alpha_{p}}$ by condition (ii). Hence, in particular, $\sum_{k=0}^{l-1}{\overline{\lambda_{j}}}^{k} y_{n_{0}+k}=0$. This shows that $D_{j}=0$ for each $j \in\{w+1, \ldots, l\}$. Thus $\operatorname{det} G_{j}=0$ and therefore also $A_{j}^{(1)}=0$ for $w+1 \leq j \leq l$. In view of (5.3) this shows the ultimate periodicity of $\left(x_{n}\right)_{n \in \mathbb{N}}$ and we are done.

We will now show the necessity of the conditions (i) and (ii).

(i) Since $\mathbf{r} \odot \mathbf{s} \in \mathcal{D}_{d+q}$ the second inclusion in (1.2) implies that $1 \geq$ $\rho(R(\mathbf{r} \odot \mathbf{s})) \geq \rho(R(\mathbf{s}))$. Since $s_{0} \neq 0, \mathbf{s}$ is related to a polynomial over $\mathbb{Z}$ each of whose roots are nonzero and bounded by one in modulus. This implies that each root of this polynomial is a root of unity. 
Still we have to show that $\chi_{R(\mathbf{s})}$ has no root of multiplicity greater than 1. Suppose on the contrary that $\chi_{R(\mathbf{s})}$ has a root of multiplicity at least 2 , say $\lambda_{j_{0}}$. Let $\mathbf{x} \in \mathbb{Z}^{d+q}$ and $\left(x_{n}\right)_{n \in \mathbb{N}}:=\tau_{\mathbf{s} \odot \mathbf{r}}^{*}(\mathbf{x})$. We will show that $\left(x_{n}\right)_{n \in \mathbb{N}}$ does not end up periodically. As $\left(x_{n}\right)_{n \in \mathbb{N}}$ satisfies the recurrence relation (5.2) there are polynomials $A_{j}(1 \leq j \leq g)$ such that

$$
x_{n_{0}+k}=\sum_{j=1}^{g} A_{j}(k) \lambda_{j}^{k} .
$$

Inserting (5.2) we obtain

$$
y_{n_{0}+k}=\sum_{j=1}^{g} \sum_{h=1}^{q} s_{h} A_{j}(k+h) \lambda_{j}^{k+h} .
$$

Taking $k \in\{1, \ldots l\}$ these are $l$ equations for the $l+q$ coefficients $A_{j}^{(\nu)}$ of the polynomials $A_{j}$. However, $q$ of these $l+q$ coefficients do not occur in (5.8) because they are cancelled out in a similar way as in (5.4). One of the coefficients not occurring in (5.8) is $A_{j_{0}}^{(1)}$, as its multiplier in (5.8) is easily seen to be

$$
k \lambda_{j_{0}}^{k} \chi_{R(\mathbf{s})}\left(\lambda_{j_{0}}\right)+\lambda_{j_{0}}^{k+1} \chi_{R(\mathbf{s})}^{\prime}\left(\lambda_{j_{0}}\right) .
$$

This vanishes as $\lambda_{j_{0}}$ is a double zero of $\chi_{R(\mathbf{s})}$. Thus we use (5.8) in order to calculate $l$ of the $l+q$ coefficients $A_{j}^{(\nu)}$. Each $q$-tuple $z_{1}, \ldots, z_{q}$ of integers yields a system of $q$ equations. Indeed,

$$
z_{k}=\sum_{j=1}^{g} A_{j}(k) \lambda_{j}^{k} \quad(1 \leq k \leq q) .
$$

This system can be used in order to calculate the remaining $q$ coefficients $A_{j}^{(\nu)}$, among which we have $A_{j_{0}}^{(1)}$. We choose $z_{1}, \ldots, z_{q}$ in a way that $A_{j_{0}}^{(1)} \neq 0$. This choice gives us values for all coefficients $A_{j}^{(\nu)}$.

For $k \geq q$ define the integers $z_{k}$ by

$$
z_{k}:=\sum_{j=1}^{g} A_{j}(k) \lambda_{j}^{k} .
$$

Then by $(5.2)$ the sequence $\tau_{\mathbf{s} \odot \mathbf{r}}^{*}\left(\left(z_{0}, \ldots, z_{d+l-1}\right)\right)$ obviously satisfies the recurrence relation $\sum_{i=0}^{q} s_{i} z_{n_{0}+k+i}=\sum_{i=0}^{q} s_{i} z_{n_{0}+k+l+i}$. As $A_{j_{0}}^{(1)} \neq 0$ this sequence does not end up periodically by Lemma 5.1, a contradiction.

(ii) Since $\mathbf{r} \odot \mathbf{s} \in \mathcal{D}_{d+q},\left(x_{n}\right)_{n \in \mathbb{N}}$ in (5.3) is ultimately periodic. By Lemma 5.1 this implies that $A_{j}^{(1)}=0$ for each $j \in\{w+1, \ldots, l\}$. 
The proof of the sufficiency of the three conditions shows that this is equivalent to $D_{j}=0$ and thus to the fact that (5.7) holds for $\alpha_{1}, \ldots, \alpha_{b}$. As the latter is just a reformulation of (ii) we are done.

We continue with the short proof of Proposition 5.1. It is then clear how to deduce the other propositions from Theorem 5.1.

Proof of Proposition 5.1. Set $\mathbf{s}=(-1)$ and note that $\chi_{R(\mathbf{s})}=\Phi_{1}$. Then an application of Theorem 5.1 immediately shows the result.

The following corollary gives a criterion which ensures that certain points do not belong to $\mathcal{D}_{d}^{(p)}$.

Corollary 5.1. If $\Phi_{p} \mid \chi_{R(\mathbf{r})}$ then $\mathbf{r} \notin \mathcal{D}_{d}^{(p)}$.

Proof. Suppose on the contrary that $\mathbf{r} \in \mathcal{D}_{d}^{(p)}$. We will derive a contradiction. Let $q:=\varphi(p)$ where $\varphi$ denotes Euler's totient function. Define $\mathbf{s} \in \mathbb{Z}^{q}$ by the equation $\chi_{R(\mathbf{s})}=\Phi_{p}$. Then by Theorem 5.1 we have $\mathbf{r} \odot \mathbf{s} \in \mathcal{D}_{d+q}$. On the other hand, since $\Phi_{p} \mid \chi_{R(\mathbf{r})}$, there exists $\mathbf{r}^{\prime} \in \mathbb{R}^{d-q}$ such that $\mathbf{r}=\mathbf{r}^{\prime} \odot \mathbf{s}$. As $\mathbf{r} \in \mathcal{D}_{d}$ Theorem 5.1 implies that $\mathbf{r}^{\prime} \in \mathcal{D}_{d-q}^{(p)}$. Thus we have $\mathbf{r}^{\prime} \odot(\mathbf{s} \odot \mathbf{s})=\mathbf{r} \odot \mathbf{s} \in \mathcal{D}_{d+q}$. However, because $\mathbf{r} \in \mathcal{D}_{d}^{(p)}$ and $\chi_{R(\mathbf{s} \odot \mathbf{s})}=\Phi_{p}^{2}$ is not the product of pairwise disjoint cyclotomic polynomials this contradicts Theorem 5.1.

\section{Algorithms and concrete results}

6.1. An algorithm for describing $\mathcal{D}_{d}^{(p)}$. After the formal introduction of the sets $\mathcal{D}_{d}^{(p)}$ in (4.1) we will present a way for characterizing these sets algorithmically. For $p=0$ an algorithm can be found in [1] (see also $[4,19,20])$. We first give a brief outline of this algorithm and afterwards we apply similar ideas in order to generalize it for other values of $p$.

Definition. Let $\mathbf{r} \in \mathcal{D}_{d}$. A set $\mathcal{V} \subset \mathbb{Z}^{d}$ that satisfies

(1) $\forall \mathbf{x} \in \mathbb{Z}^{d} \exists k \in \mathbb{N},\left(\mathbf{b}_{1}, \ldots, \mathbf{b}_{k}\right) \in \mathcal{V}^{k}: \mathbf{x}=\sum_{j=1}^{k} \mathbf{b}_{j}$,

(2) $\mathbf{x} \in \mathcal{V} \Rightarrow \tau_{\mathbf{r}}(\mathbf{x}),-\tau_{\mathbf{r}}(-\mathbf{x}) \in \mathcal{V}$

is called a set of witnesses of $\mathbf{r}$.

Such a set exists and can be chosen to be finite because the linear part of the mapping $\tau_{\mathbf{r}}$ is contractive. We refer to [1], where details are discussed.

A set of witnesses has nice properties concerning $\tau_{\mathbf{r}}$.

Theorem 6.1 (cf. [1, Theorem 5.1]). Let $\mathbf{r} \in \mathcal{D}_{d}$ and let $\mathcal{V}$ be a set of witnesses of $\mathbf{r}$. We have $\mathbf{r} \in \mathcal{D}_{d}^{(0)}$ if and only if $\mathcal{V}$ does not contain purely periodic points with respect to $\tau_{\mathbf{r}}$ except $\mathbf{0}$. 
The definition of the set of witnesses can be extended to sets $Q \subset \mathcal{D}_{d}$.

Definition. Let $Q \subset \mathcal{D}_{d}$. A set $\mathcal{V} \subset \mathbb{Z}^{d}$ that satisfies

(1) $\forall \mathbf{x} \in \mathbb{Z}^{d} \exists k \in \mathbb{N},\left(\mathbf{b}_{1}, \ldots, \mathbf{b}_{k}\right) \in \mathcal{V}^{k}: \mathbf{x}=\sum_{j=1}^{k} \mathbf{b}_{j}$,

(2) $\tau_{\mathbf{r}}(\mathcal{V}) \cup-\tau_{\mathbf{r}}(-\mathcal{V}) \subset \mathcal{V}$ holds for all $\mathbf{r} \in Q$

is called a set of witnesses of $Q$.

Such a set exists and can be chosen to be finite provided that the diameter of $Q$ is small. For details we refer again to [1].

Further define the following graph.

Definition. For a finite set $W \subset \mathbb{Z}^{d}$ and a set $Q \subset \mathcal{D}_{d}$, we define $G(W, Q)=$ $(V, E)$ to be the smallest directed graph with vertices $V \subset \mathbb{Z}^{d}$ and edges $E \subset \mathbb{Z}^{d} \times \mathbb{Z}^{d}$ such that

(1) $W \subseteq V$,

(2) $\tau_{\mathbf{r}}(V) \subset V$ for all $\mathbf{r} \in Q$,

(3) $E=\left\{\left(\mathbf{x}, \tau_{\mathbf{r}}(\mathbf{x})\right) \mid \mathbf{x} \in V, \mathbf{r} \in Q\right\}$.

We are interested in the (directed, simple) cycles of such a graph, i.e., in paths of the form $\mathbf{v}_{0} \rightarrow \mathbf{v}_{1} \rightarrow \ldots \rightarrow \mathbf{v}_{l-1} \rightarrow \mathbf{v}_{0}$ with pairwise disjoint $\mathbf{v}_{i}$ $(0 \leq i \leq l-1)$. To avoid confusion with cycles of $\tau_{\mathbf{r}}$ we will refer to cycles of graphs as graph-cycles although the two types of cycles are closely related to each other. A graph-cycle of length $l$ consists of $l d$-dimensional integer vectors. By the definition of the edges a graph-cycle has the shape

$$
\left(x_{0}, \ldots, x_{d-1}\right) \rightarrow\left(x_{1}, \ldots, x_{d}\right) \rightarrow \cdots \rightarrow\left(x_{l-1}, \ldots, x_{d-2}\right) \rightarrow\left(x_{0}, \ldots, x_{d-1}\right) .
$$

Similar to a cycle of $\tau_{\mathbf{r}}$, a graph-cycle is uniquely determined by the $l$ integers $x_{0}, \ldots, x_{l-1}$. Again the elements are ordered cyclically. This enables us to identify a graph-cycle of $G(\mathcal{V}, Q)$ for some sets $\mathcal{V}$ and $Q$ with the integer sequence of corresponding length that determines it and denote it also by $\left\langle x_{0}, \ldots, x_{l-1}\right\rangle$.

Finally, for some graph-cycle $\left\langle x_{0}, \ldots, x_{l-1}\right\rangle$, consider the system of $l$ double inequalities

$$
0 \leq r_{0} x_{j}+\cdots+r_{d-1} x_{j+d-1}+x_{j+d}<1, \quad(0 \leq j<l)
$$

where the indices of the $x_{i}$ have to be taken modulo $l$, and denote $P_{d}\left(\left\langle x_{0}, \ldots, x_{l-1}\right\rangle\right):=\left\{\left(r_{0}, \ldots, r_{d-1}\right) \in \mathbb{R}^{d} \mid\left(r_{0}, \ldots, r_{d-1}\right)\right.$ satisfies $\left.(6.1)\right\}$. Note that a graph-cycle $\mathcal{B}$ is a cycle of $\tau_{\mathbf{r}}$ for some $\mathbf{r} \in \mathcal{D}_{d}$ if and only if $P_{d}(\mathcal{B}) \neq \emptyset$. More precisely,

$$
\mathbf{r} \in P_{d}\left(\left\langle x_{0}, \ldots, x_{l-1}\right\rangle\right) \Longleftrightarrow\left\langle x_{0}, \ldots, x_{l-1}\right\rangle \in C(\mathbf{r}) .
$$

It is possible to characterize $Q \cap \mathcal{D}_{d}^{(0)}$ by using a corresponding set of witnesses $\mathcal{V}$ provided that it is finite. This characterization is based on an idea of Brunotte (see [7]). 
Theorem 6.2 (Brunotte Algorithm, cf. [1, Theorem 5.2]). Let $Q \subset \mathcal{D}_{d}$ and suppose that $\mathcal{V}$ is a finite set of witnesses of $Q$. Furthermore denote by $\Pi$ the set of graph-cycles of $G(\mathcal{V}, Q)$ without the trivial one $\langle 0\rangle$. Then

$$
\mathcal{D}_{d}^{(0)} \cap Q=Q \backslash \bigcup_{\pi \in \Pi} P_{d}(\pi) .
$$

Our aim is to adapt Theorem 6.1 and Theorem 6.2 in order to get algorithms for describing $\mathcal{D}_{d}^{(p)}$ for $p \neq 0$. The main problem is that even though a set of witnesses of a set $Q$ (where $Q$ may consist of one point only) contains enough cycles to completely characterize $Q \cap \mathcal{D}_{d}^{(0)}$ it may not include all cycles of $\tau_{\mathbf{r}}$ with $\mathbf{r} \in Q$.

Suppose that $p \neq 0$ and $\mathbf{r} \in \mathcal{D}_{d}$. For a given set of witnesses of $\mathbf{r}$ we have the following situation. When $\mathbf{0}$ is the only purely periodic point of $\tau_{\mathbf{r}}$ then $\mathbf{r} \in \mathcal{D}_{d}^{(0)}$ by Theorem 6.1 and therefore, as $\mathcal{D}_{d}^{(0)} \subset \mathcal{D}_{d}^{(p)}$, we immediately conclude that $\mathbf{r} \in \mathcal{D}_{d}^{(p)}$. If the set of witnesses includes other purely periodic points than $\mathbf{0}$ then these points induce a set $\Pi$ of cycles. If this set contains a cycle $\mathcal{B}$ with $S_{p}(\mathcal{B}) \neq 0$ then $\mathbf{r} \notin \mathcal{D}_{d}^{(p)}$ and we are done. A problem occurs if $\Pi \neq\{(0)\}$ and $S_{p}(\mathcal{B})=0$ for all $\mathcal{B} \in \Pi$ since we do not know whether there exists a cycle $\mathcal{B}^{\prime}$ with $\mathcal{B}^{\prime} \notin \Pi$ and $S_{p}\left(\mathcal{B}^{\prime}\right) \neq 0$.

The following lemma will show that we can use the idea of the set of witnesses even for analyzing the structure of $\mathcal{D}_{d}^{(p)}$, however, with some additional expenditure.

Lemma 6.1. Let $\mathbf{r} \in \mathcal{D}_{d}$ and $\mathcal{V}$ a set of witnesses of $\mathbf{r}$. If $\mathbf{x} \in \mathbb{Z}^{d}$ is purely periodic w.r.t. $\tau_{\mathbf{r}}$ then there exist integer points $\mathbf{z}_{1}, \ldots, \mathbf{z}_{n} \in \mathcal{V}$ such that $\sum_{j=1}^{n} \mathbf{z}_{j}=\mathbf{x}$ and $\sum_{j=1}^{k} \mathbf{z}_{j}$ is purely periodic (w.r.t. $\tau_{\mathbf{r}}$ ) for all $k \in\{1, \ldots, n\}$.

Proof. Let $\mathbf{y}$ be an arbitrary purely periodic point with respect to $\tau_{\mathbf{r}}$ having period $l$. For proving the lemma it suffices to show that we can find $\mathbf{w}_{1}, \ldots, \mathbf{w}_{m} \in \mathcal{V}$ such that $\mathbf{y}=\sum_{j=1}^{m} \mathbf{w}_{j}$ and $\sum_{j=1}^{m-1} \mathbf{w}_{j}$ is purely periodic.

By Definition $6.1 \mathbf{y}$ can be represented as

$$
\mathbf{y}=\sum_{j=1}^{m} \mathbf{w}_{j}^{\prime}
$$

with $\mathbf{w}_{1}^{\prime}, \ldots, \mathbf{w}_{m}^{\prime} \in \mathcal{V}$. Let $q \in \mathbb{N}$. Then $q$ applications of $\tau_{\mathbf{r}}^{l}$ yield

$$
\tau_{\mathbf{r}}^{l q}(\mathbf{y})=\mathbf{y}=\tau_{\mathbf{r}}^{l q}\left(\sum_{j=1}^{m-1} \mathbf{w}_{j}^{\prime}\right)+\mathbf{w}_{m}^{\prime \prime}
$$

with $\mathbf{w}_{m}^{\prime \prime} \in \mathcal{V}$ by the construction of the set of witnesses. Since $\mathbf{r} \in \mathcal{D}_{d}$ we can choose $q$ such that $\tau_{\mathbf{r}}^{l q}\left(\sum_{j=1}^{m-1} \mathbf{w}_{j}^{\prime}\right)$ is a purely periodic point of $\tau_{\mathbf{r}}$. On 
the other hand, again by the construction of the set of witnesses, we have

$$
\tau_{\mathbf{r}}^{l q}\left(\sum_{j=1}^{m-1} \mathbf{w}_{j}^{\prime}\right)=\sum_{j=1}^{m-1} \mathbf{w}_{j}^{\prime \prime}
$$

for some $\mathbf{w}_{1}^{\prime \prime}, \ldots, \mathbf{w}_{m-1}^{\prime \prime} \in \mathcal{V}$. Setting $\mathbf{w}_{j}:=\mathbf{w}_{j}^{\prime \prime}$ for all $j \in\{0, \ldots, m\}$ shows the claim.

We first show how Lemma 6.1 can be used to check whether a given point $\mathbf{r} \in \mathcal{E}_{d}$ is contained in $\mathcal{D}_{d}^{(p)}$ or not. After that we will generalize this to sets $Q \subset \mathcal{E}_{d}$.

Theorem 6.3. Let $p \in \mathbb{N}, \mathbf{r} \in \mathcal{E}_{d}$ and $\mathcal{V}$ a finite set of witnesses of $\mathbf{r}$. If there exists a set $W \subset \mathbb{Z}^{d}$ with

- $\mathbf{0} \in W$,

- $\mathcal{V}+Z \subset W$,

where $Z$ consists of all purely periodic points (w.r.t $\tau_{\mathbf{r}}$ ) contained in $W$, such that for each cycle $\mathcal{B}$ generated by elements of $W$ we have $S_{p}(\mathcal{B})=0$, then $\mathbf{r} \in \mathcal{D}_{d}^{(p)}$.

Proof. The theorem is a direct consequence of Lemma 6.1.

Note that by [1, Theorem 5.1] there always exists a finite set of witnesses whenever $\mathbf{r} \in \mathcal{E}_{d}$. Theorem 6.3 provides an algorithm for verifying whether $\mathbf{r} \in \mathcal{D}_{d}^{(p)}$ or not. We can now construct a set $W$ that satisfies the conditions of Theorem 6.3 as follows. Set $W_{0}:=\mathcal{V}$. Now, for every $k=0,1,2, \ldots$ define $W_{k}$ recursively as follows: let $\Pi_{k}$ be the set of all cycles contained in $W_{k}$ without the trivial one $\langle 0\rangle$. If $\Pi=\emptyset$ then we can stop and conclude that $\mathbf{r} \in \mathcal{D}_{d}^{(p)}$. If there exists a cycle $\mathcal{B} \in \Pi_{k}$ with $S_{p}(\mathcal{B}) \neq 0$ then we can stop, too, and we obviously have $\mathbf{r} \notin \mathcal{D}_{d}^{(p)}$. If $\Pi_{k} \neq \emptyset$ and $S_{p}(\mathcal{B})=0$ for all $\mathcal{B} \in \Pi_{k}$ then denote by $Z_{k} \subset \mathbb{Z}^{d}$ all purely periodic points induced by the cycles in $\Pi_{k}$ and let

$$
W_{k+1}:=\mathcal{V}+Z_{k}
$$

It can be shown easily by induction on $k$ that, whenever $W_{k+1}$ contains a purely periodic point, $W_{k+1}$ contains all purely periodic points of the corresponding cycle. We also see by Lemma 6.1 that this procedure terminates at some stage $k=k_{0}$ since $C(\mathbf{r})$ is finite for $\mathbf{r} \in \mathcal{E}_{d}$. This leads to a set $W:=W_{k_{0}}$ satisfying the conditions of Theorem 6.3 in the relevant case.

Finally we are going to generalize the results from above in order to characterize $Q \cap \mathcal{D}_{d}^{(p)}$ for some $Q \subset \mathcal{E}_{d}$ using Theorem 6.2.

Theorem 6.4. Let $p \in \mathbb{N}, Q \subset \mathcal{E}_{d}$ closed and $\mathcal{V}$ a finite set of witnesses of $Q$. Denote by $W \subset \mathbb{Z}^{d}$ a finite set with

- $\mathbf{0} \in W$, 
- $\mathcal{V}+Z \subset W$,

where $Z$ consists of all purely periodic points w.r.t. $\tau_{\mathbf{r}}$ for some $\mathbf{r} \in Q$ contained in $W$. Then

$$
Q \cap \mathcal{D}_{d}^{(p)}=Q \backslash \bigcup_{\mathcal{B} \in \Pi, S_{p}(\mathcal{B}) \neq 0} P_{d}(\mathcal{B})
$$

where $\Pi$ consists of the graph-cycles of $G(W, Q)$ except $\langle 0\rangle$.

Proof. The theorem is a direct consequence of Lemma 6.1.

By [1, Theorem 5.2] (see also [19, Lemma 2.2]) there exists a finite set of witnesses of closed $Q \subset \mathcal{E}_{d}$ provided that $Q$ is sufficiently small. To construct $W$ again set $W_{0}:=\mathcal{V}$. Now, for every $k=0,1,2, \ldots$, let $\Pi_{k}$ the set of all graph-cycles of $G\left(W_{k}, Q\right)$ (without the trivial one $\langle 0\rangle$ ) that are cycles for some $\mathbf{r} \in Q$. If

$$
\left\{\mathcal{B} \in \Pi_{k} \backslash \Pi_{k-1} \mid S_{p}(\mathcal{B})=0\right\}=\emptyset
$$

we stop and have

$$
Q \cap \mathcal{D}_{d}^{(p)}=Q \backslash \bigcup_{\mathcal{B} \in \Pi_{k}, S_{p}(\mathcal{B}) \neq 0} P_{d}(\mathcal{B}) .
$$

If (6.2) does not hold let $Z_{k} \subset \mathbb{Z}^{d}$ all purely periodic points induced by the cycles of $\Pi_{k}$ and let

$$
W_{k+1}:=\mathcal{V}+Z_{k}
$$

Again this process terminates at some level $k=k_{0}$. Setting $W:=W_{k_{0}}$ yields a set $W$ satisfying the conditions of the theorem.

6.2. The behavior of $\tau_{\mathrm{r}}$ on the boundary of $\mathcal{E}_{3}$. We now apply the algorithms of the last subsection in order to describe $\mathcal{D}_{2}^{(p)}$ for $p \in\{1,2\}$ and $\mathcal{D}_{1}^{(p)}$ for $p \in\{3,4,6\}$. Together with the results of Section 5 this will give us a characterization of the parameters $\mathbf{r} \in \partial \mathcal{E}_{3}$ for which $\tau_{\mathbf{r}}$ has only periodic orbits, i.e., a characterization of the set $\mathcal{D}_{3} \backslash \mathcal{E}_{3}$. As one can easily derive from the results in Section $3, \partial \mathcal{E}_{3}=\partial \mathcal{D}_{3}$ consists of the two triangles $E_{3}^{(-1)}$ and $E_{3}^{(1)}$ and of the surface $E_{3}^{(\mathbb{C})}$. In particular, from (2.4) we easily see that

$$
\begin{aligned}
E_{3}^{(1)} & =\{(s, s+t+s t, s t+t+1) \mid-1 \leq s, t \leq 1\} \quad \text { and } \\
E_{3}^{(-1)} & =\{(-s, s-t-s t, s t+t-1) \mid-1 \leq s, t \leq 1\} .
\end{aligned}
$$

The periodic parameters in $E_{3}^{(-1)}$ (i.e., the parameters $\mathbf{r}$ for which $\tau_{\mathbf{r}}(\mathbf{z})$ is ultimately periodic for each starting value $\mathbf{z} \in \mathbb{Z}^{3}$ ) can be described via the set $\mathcal{D}_{2}^{(1)}$ by Proposition 5.1. According to Lemma $4.1, \mathcal{D}_{2}^{(1)}$ is equal to $\mathcal{D}_{2}^{(0)}$ apart from few exceptional points on the boundary. Thus we can use the 

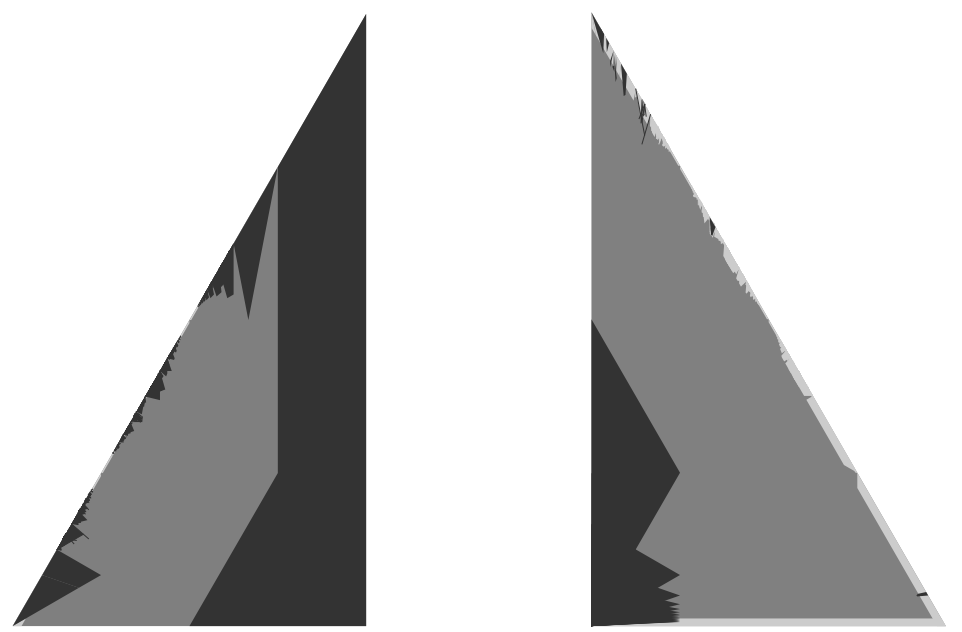

Figure 6.1. The triangles $E_{3}^{(-1)}$ (left hand side) and $E_{3}^{(1)}$ (right hand side). The dark grey regions correspond to parameters $\mathbf{r}$ for which $\tau_{\mathbf{r}}$ is ultimately periodic for each starting value $\mathbf{z} \in \mathbb{Z}^{3}$. For the parameters $\mathbf{r}$ in the black regions there exists at least one starting value $\mathbf{z} \in \mathbb{Z}^{3}$ such that the orbit $\left(\tau_{\mathbf{r}}^{k}(\mathbf{z})\right)_{k \in \mathbb{N}}$ becomes unbounded. The light grey regions are not yet characterized.
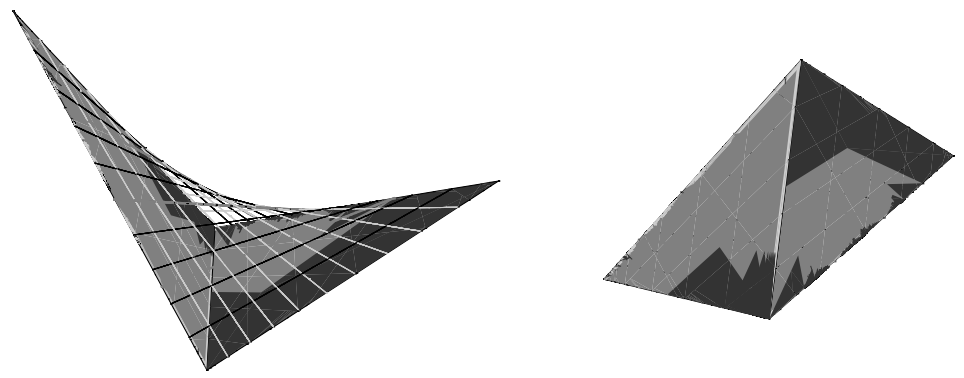

Figure 6.2. The boundary of $\mathcal{D}_{3}$. On the left hand side we see a view of $\mathcal{D}_{3}$ where the surface $E_{3}^{(\mathbb{C})}$ is transparent. Some lines of it can be characterized with help of Propositions 5.3 to 5.5. On the right hand side another view of $\mathcal{D}_{3}$ is presented. Here we see the two triangles $E_{3}^{(-1)}$ and $E_{3}^{(1)}$. The colors have the following meaning. Black: there exist unbounded orbits; dark grey: all orbits are ultimately periodic; light grey: not yet characterized. 
description of the set $\mathcal{D}_{2}^{(0)}$ which has been studied extensively ( $c f$. e.g. [4]) in order to get the periodic parameters of large parts of $E_{3}^{(-1)}$.

The periodic parameters in $E_{3}^{(1)}$ can be described via the set $\mathcal{D}_{2}^{(2)}$ by Proposition 5.2. Since large parts of $\mathcal{D}_{2}^{(2)}$ can be characterized with help of the algorithms presented in the last subsection we obtain a description of the periodic parameters of large parts of $E_{3}^{(1)}$.

Finally, small parts of $E_{3}^{(\mathbb{C})}$ can be characterized by using Propositions 5.3 to 5.5 . Indeed they yield straight lines on the surface $E_{3}^{(\mathbb{C})}$ which belong to $\mathcal{D}_{3}$.

The results of the algorithmic description of the periodic orbits of $\partial \mathcal{E}_{3}$ are visualized in Figures 6.1 and 6.2. The pictures suggest that the set of parameters $\mathbf{r} \in \partial \mathcal{E}_{d}$ that give rise to periodic orbits of $\tau_{\mathbf{r}}$ has a very complicated structure.

\section{References}

[1] S. Akiyama, T. Borbély, H. Brunotte, A. Pethő, and J. M. Thuswaldner, Generalized radix representations and dynamical systems. I. Acta Math. Hungar. 108 (2005), 207-238.

[2] S. Akiyama, H. Brunotte, A. Pethő, And W. Steiner, Remarks on a conjecture on certain integer sequences. Period. Math. Hungar. 52 (2006), 1-17.

[3] — Periodicity of certain piecewise affine planar maps. Tsukuba J. Math. 32 (2008), $197-251$.

[4] S. Akiyama, H. Brunotte, A. Pethő, and J. M. Thuswaldner, Generalized radix representations and dynamical systems. II. Acta Arith. 121 (2006), 21-61.

[5] S. Akiyama, C. Frougny, And J. Sakarovitch, Powers of rationals modulo 1 and rational base number systems. Israel J. Math. 168 (2008), 53-91.

[6] D. W. Boyd, The beta expansion for Salem numbers. In Organic mathematics (Burnaby, BC, 1995), vol. 20 of CMS Conf. Proc. Amer. Math. Soc., Providence, RI, 1997, 117-131.

[7] H. Brunotte, On trinomial bases of radix representations of algebraic integers. Acta Sci. Math. (Szeged) 67 (2001), 521-527.

[8] A. T. FAM, The volume of the coefficient space stability domain of monic polynomials. Proc. IEEE Int. Symp. Circuits and Systems 2 (1989), 1780-1783.

[9] A. T. Fam And J. S. Meditch, A canonical parameter space for linear systems design. IEEE Trans. Autom. Control 23 (1978), 454-458.

[10] C. Frougny and B. Solomyak, Finite beta-expansions. Ergodic Theory Dynam. Systems 12 (1992), 713-723.

[11] A. Huszti, K. Scheicher, P. Surer, And J. M. Thuswaldner, Three-dimensional symmetric shift radix systems. Acta Arith. 129 (2007), 147-166.

[12] P. Kirschenhofer, A. Pethő, And J. M. Thuswaldner, On a family of three term nonlinear integer recurrences. Int. J. Number Theory 4 (2008), 135-146.

[13] E. Lehmer, On the magnitude of the coefficients of the cyclotomic polynomial. Bull. Amer. Math. Soc. 42 (1936), 389-392.

[14] J. Lowenstein, S. Hatjispyros, And F. Vivaldi, Quasi-periodicity, global stability and scaling in a model of Hamiltonian round-off. Chaos 7 (1997), 49-66.

[15] A. Ретнö, On a polynomial transformation and its application to the construction of a public key cryptosystem. In Computational number theory (Debrecen, 1989), de Gruyter, Berlin, 1991, 31-43.

[16] - On the boundary of the closure of the set of contractive polynomials. Integers 9 (2009), $311-325$. 
[17] K. Schmidt, On periodic expansions of Pisot numbers and Salem numbers. Bull. London Math. Soc. 12 (1980), 269-278.

[18] I. Schur, Über Potenzreihen, die im Innern des Einheitskreises beschränkt sind. II. J. reine und angew. Math 148 (1918), 122-145.

[19] P. Surer, Characterisation results for shift radix systems. Math. Pannon. 18 (2007), 265297.

[20] - $\_$-shift radix systems and radix representations with shifted digit sets. Publ. Math. (Debrecen) $\mathbf{7 4}$ (2009), 19-43.

Peter Kirschenhofer

Chair of Mathematics and Statistics

University of Leoben

Franz-Josef-Str. 18

A-8700 Leoben, AUSTRIA

E-mail: peter.kirschenhofer@mu-leoben.at

Attila Peтнö

Faculty of Informatics

Number Theory Research Group

Hungarian Academy of Sciences and University of Debrecen

P.O. Box 12

H-4010 Debrecen, HUNGARY

E-mail: Petho.Attila@inf.unideb.hu

Paul Surer

Departamento de Matemática

IBILCE - UNESP

Rua Cristóvão Colombo, 2265 - Jardim Nazareth

15.054-000 São José do Rio Preto - SP, BRAZIL

E-mail: me@palovsky.com

Jörg ThuswaldneR

Chair of Mathematics and Statistics

University of Leoben

Franz-Josef-Str. 18

A-8700 Leoben, AUSTRIA

E-mail: joerg.thuswaldner@unileoben.ac.at 